\title{
Energy Consumption Of Visual Sensor Networks: Impact Of Spatio-Temporal Coverage
}

\author{
Alessandro Redondi, Dujdow Buranapanichkit, Matteo Cesana, Marco Tagliasacchi and \\ Yiannis Andreopoulos
}

\begin{abstract}
Wireless visual sensor networks (VSNs) are expected to play a major role in future IEEE 802.15.4 personal area networks (PAN) under recently-established collision-free medium access control (MAC) protocols, such as the IEEE 802.15.4e-2012 MAC. In such environments, the VSN energy consumption is affected by the number of camera sensors deployed (spatial coverage), as well as the number of captured video frames out of which each node processes and transmits data (temporal coverage). In this paper, we explore this aspect for uniformly-formed VSNs, i.e., networks comprising identical wireless visual sensor nodes connected to a collection node via a balanced cluster-tree topology, with each node producing independent identically-distributed bitstream sizes after processing the video frames captured within each network activation interval. We derive analytic results for the energyoptimal spatio-temporal coverage parameters of such VSNs under a-priori known bounds for the number of frames to process per sensor and the number of nodes to deploy within each tier of the VSN. Our results are parametric to the probability density function characterizing the bitstream size produced by each node and the energy consumption rates of the system of interest. Experimental results derived from a deployment of TelosB motes under a collision-free transmission protocol and Monte-Carlo-generated data sets reveal that our analytic results are always within $7 \%$ of the energy consumption measurements for a wide range of settings. In addition, results obtained via a multimedia subsystem (BeagleBone Linux Computer) performing differential Motion JPEG encoding and local visual feature extraction from video frames show that the optimal spatio-temporal settings derived by the proposed framework allow for substantial reduction of energy consumption in comparison to ad-hoc settings. As such, our analytic modeling is useful for early-stage studies of possible VSN deployments under collision-free MAC protocols prior to costly and time-consuming experiments in the field.
\end{abstract}

Index Terms-visual sensor networks, energy consumption, frame-rate, sensor coverage, Internet-of-Things

$\mathrm{AR}, \mathrm{MC}$ and MT are with the Dipartimento di Elettronica, Informazione e Bioingegneria, Politecnico di Milano, P.zza Leonardo da Vinci, 32 - 20133, Milano - Italy, e-mail: firstname.lastname@polimi.it. DB is with the Department of Electrical Engineering, Faculty of Engineering, Prince of Songkla University, Hat Yai, Songkla, 90112 Thailand; email: dujdow.b@psu.ac.th. YA is with the Electronic and Electrical Engineering Department, University College London, Roberts Building, Torrington Place, London, WC1E 7JE, UK; tel. +442076797303; fax. +442073889325; email: i.andreopoulos@ucl.ac.uk.

AR, MC and MT acknowledge the financial support of the Future and Emerging Technologies (FET) programme within the Seventh Framework Programme for Research of the European Commission, under FETOpen grant number: 296676. YA was funded by the UK EPSRC, grant EP/K033166/1.

This paper appears in the IEEE Trans. Circ. and Syst. for Video Technol., 2014. Copyright (c) 2014 IEEE. Personal use of this material is permitted. However, permission to use this material for any other purposes must be obtained from the IEEE by sending a request to pubspermissions@ieee.org.

\section{INTRODUCTION}

The integration of low-power wireless networking technologies such as IEEE 802.15.4-enabled transceivers with inexpensive camera hardware has enabled the development of the so-called visual sensor networks (VSNs) [1]. VSNs can be thought of as networks of wireless devices capable of sensing multimedia content, such as still images and video, audio, depth maps, etc. Via the recent provisioning of an allIPv6 network layer under 6LoWPAN [2] and the emergence of collision-free low-power medium access control (MAC) protocols, such as the time slotted channel hopping (TSCH) of IEEE 802.15.4e-2012 [3], VSNs are expected to play a major role in the Internet-of-Things (IoT) paradigm [4], [5].

\section{A. Review of Visual Sensor Networks}

In comparison to traditional wireless sensor networks, VSNs are uniquely challenging because of their heavy computational and bandwidth requirements that stretch hardware and networking infrastructures to their limits. Hence, an increasing number of VSN solutions were proposed recently, focusing on: new transmission protocols allowing for high-bandwidth collision-free communications [6] [7], in-network processing techniques $[\overline{8}]$ and optimized multimedia processing [9]. Also, several hardware solutions have been proposed, with the aim of finding a VSN platform that could be used for a broad range of multimedia tasks [10][12].

Most of these proposed hardware solutions can be abstracted as two tightly-coupled subsystems, shown in Figure 1.b): a multimedia processor board and a low-power radio subsystem [13]-[15], interconnected via a push model. Within each node of the VSN, the multimedia subsystem is responsible for acquiring images, processing them and pushing the processed visual data to the radio subsystem, which transmits it to a remote location. For example, in a traditional surveillance application, the multimedia subsystem would compress or process (e.g., extract visual features [16], [17]) the acquired images and push the resulting bitstream to the radio subsystem for transmission to a central controller, where the data would be analyzed or stored.

Similar to traditional wireless sensor networks, VSN nodes are usually battery operated. Hence, energy consumption plays a crucial role in the design of a VSN, especially for those applications where a VSN is required to operate for days or even weeks without external power supply. In 

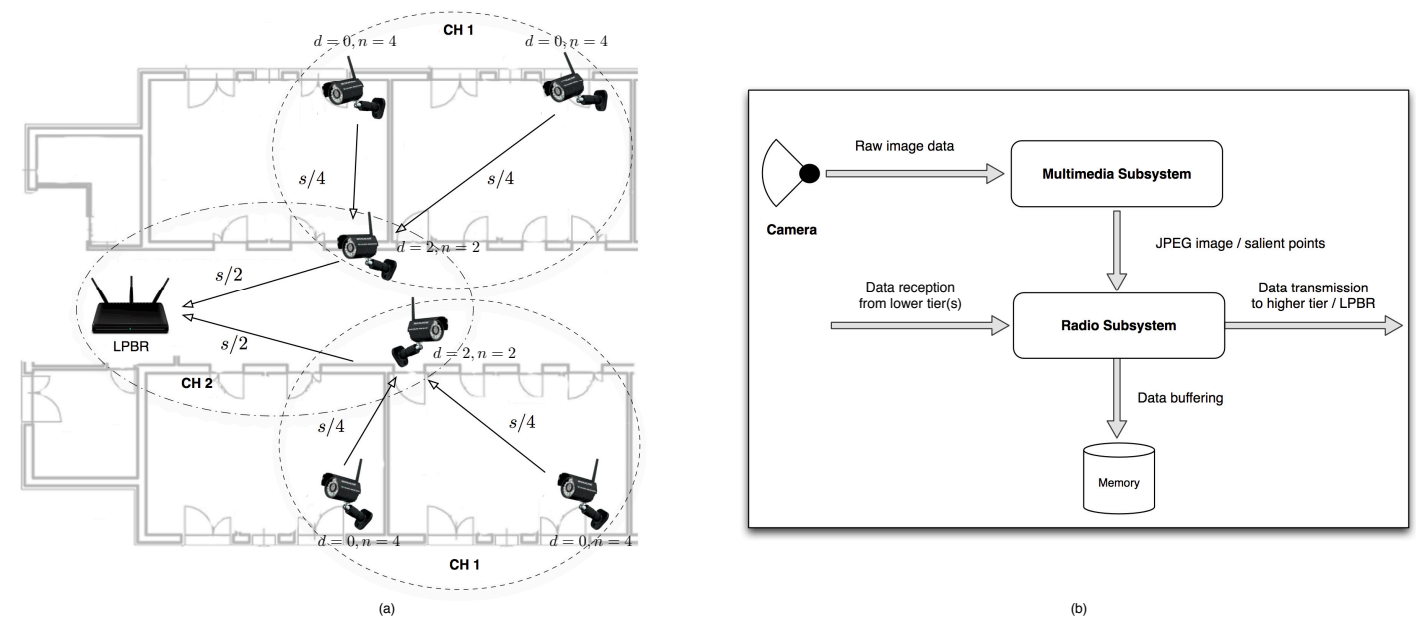

Figure 1. (a) Two-tier uniformly-formed cluster-tree topology in a visual sensor network for surveillance, where every visual sensor (video camera) has its own spatial coverage (and different channels are used within the indicated ellipses), with $s$ indicating the bits consumed by each receiver/relay node within each active interval of $T$ seconds. (b) Detail of the camera node system: each node comprises a multimedia subsystem and a radio subsystem. If required, each node can buffer parts of its data stream for later transmission.

the last few years, several proposals strive for lifetime maximization in VSNs. Specifically, solutions are available for energy-aware protocols [10], [18], cross-layer optimization [19], application tradeoffs [20] and deployment strategies [21]. While existing work addresses transmission, scheduling and protocol design aiming for energy efficiency, it does not consider the impact of the spatio-temporal coverage in the energy consumption of VSNs. This is precisely the focus of this paper.

\section{B. Scenario}

We consider wireless visual sensor networks comprising a cluster-tree topology, such as the one illustrated in Figure 1.(a), where each camera node processes and transmits visual data to the nodes of the higher tier, or to the Low-Power Border Router (LPBR) [2] that can relay the streams to any IP address over the Internet for analysis and processing. Moreover, we focus on the case of a uniformly-formed VSN, i.e. a network of identical sensor nodes that, within each activation interval, are: $(i)$ producing bitstream sizes with the same statistical characterization and (ii) connected to the base station via a balanced clustertree topology [22], represented by a symmetric and acyclic graph with balanced bandwidth allocation per link. Each node also relays streams stemming from $d$ other nodes of lower tier(s). Within each node, the multimedia and radio subsystems work in parallel [Figure 1 (b)]: while the multimedia system acquires and processes data corresponding to the current video frame, the radio subsystem transmits (or relays) the multimedia stream stemming from the processing of previous video frame(s).

Let $\frac{s}{T}$ kilobit-per-second (kbps) be the average bandwidth at each node (in transmit or receive mode), with $s$ indicating the bits consumed by each receiver/relay node over the VSN active interval of $T$ seconds. For example, for a 802.15.4compliant VSN and $T=1$ second, the average consumption rate would be $250 \mathrm{kbps}$ at the physical layer. The MAC layer of the network is operating under a collision-free timedivision (or time-frequency division) multiple access [3], [6], [7], [18], [23], so that each tier in the network can be configured in a way that simultaneous transmissions in the same channel are avoided. The number of frames captured by each camera during the operational time interval of the VSN, i.e. each node's temporal coverage, is controlling the frequency of the push operations. At the same time, the multimedia processing task itself (e.g., image/video compression or extraction of visual features) controls the size of the bitstream pushed to the radio subsystem within each frame's duration. On the other hand, the number of sensors in the same tier of the cluster-tree topology, i.e., the VSN's spatial coverage, and the number of nodes whose bitstreams must be relayed by each node (if any) control the bandwidth available to each sensor (i.e., its average transmission rate) in each tier under a collision-free MAC protocol. Therefore, there is a fundamental tradeoff between the spatial and temporal coverage in a network: a large number of frames leads to high bandwidth requirement per transmitter, which in turn decreases the number of sensors that can be accommodated within each tier of the VSN. Conversely, dense spatial coverage via the use of a large number of visual sensors per tier decreases the available bandwidth per sensor, which reduces the number of frames per sensor.

\section{Contribution and Paper Organization}

In this paper, we derive analytic results concerning energy-aware VSN design under the push model of Figure 1. Specifically, we are interested in the link of the aforementioned spatio-temporal tradeoff with the incurred energy consumption under well-known probability density functions modeling the pushed bitstream size of image and video applications, such as intra/inter-frame video coding and local visual features extraction and transmission, and make the following contributions: 
- We derive an analytic model that captures the expected energy consumption in function of: (i) the number of visual sensors deployed at each tier of the cluster-tree topology, (ii) the number of frames captured by each camera sensor within the operational time interval and (iii) the statistical characterization of the bitstream data volume produced by each sensor after on-board multimedia processing.

- The extrema of the derived energy consumption function are then analytically derived in order to provide closedform expressions for the minimum energy consumption of each case under consideration.

- The analytic results are validated within two applications: video coding and transmission based on differential Motion-JPEG and visual feature extraction and transmission.

While our results are directly applicable to uniformlyformed VSNs, we also indicate how they can be extended to non-uniformly formed VSNs with varying statistical characterizations for the bitstream sizes of different sensors and unbalanced bandwidth allocation for the various links of each VSN tier during each activation interval.

The rest of this paper is organized as follows: Section III presents the proposed system model, while Section III presents the theoretical results; Section IV presents realworld experiments that validate the proposed framework under controlled data production from each sensor, while Section V presents results showcasing the accuracy of the proposed model under real VSN data; finally, Section VI concludes the paper.

\section{Proposed System Model and Its Expected ENERGY CONSUMPTION}

In the following sections we introduce the components of the proposed system model. The corresponding nomenclature is summarized in Table I This sets the context for the derivation of the expected energy consumption of each node of the uniformly-formed visual sensor network in function of the utilized spatio-temporal coverage settings.

\section{A. Spatio-Temporal Coverage and Statistical Characteri- zation of Bitstream Size per VSN Node}

We consider that the visual sensor network is established under the following two application constraints:

- spatial coverage bounds; the number of deployed nodes at each tier of the cluster-tree topology, $n$, is upper- and lower-bounded, i.e. $N_{\min } \leq n \leq N_{\max }$

- temporal coverage lower bound; the total frame acquisitions, $k$, within a pre-defined time interval, $T$, is lowerbounded, i.e. $k \geq K_{\min }$

The bounds of the spatio-temporal coverage stem from application specifics, such as: the cost of installing and maintaining visual sensors, the minimum and maximum spatial coverage required for the area to be monitored, and the minimum number of frames that allows for visual data gathering and analysis with sufficient temporal resolution within $T$ seconds.
Since the multimedia subsystem of each visual sensor produces varying amounts of data depending on the monitored events and the specifics of the visual analysis and processing under consideration, the bitstream size produced by each sensor node in such multimedia applications is a non-deterministic quantity. Therefore, the bitstream size produced when each visual node processes $k$ frames within an activation interval is a random variable $(\mathrm{RV}), \mathcal{X}_{k}$, characterized by its probability density function (PDF), $P\left(\chi_{k}\right)$, $\mathcal{X}_{k} \sim P\left(\chi_{k}\right)$. Since the underlying processes deriving this bitstream may not be stationary and/or this data may include multi-rate channel codes (or retransmissions) to alleviate channel impairments due to propagation and other environmental effects of transmission, we assume marginal statistics for $P\left(\chi_{k}\right)$, which are derived starting from a doubly-stochastic model for the multimedia processing. Specifically, such marginal statistics can be obtained by [24], [25]: (i) fitting PDFs to sets of past measurements of bitstream sizes transmitted by each sensor, with the statistical moments (parameters) of such distributions characterized by another PDF; (ii) integrating over the parameter space to derive the final form of $P\left(\chi_{k}\right)$. For example, if the bitstream size is modeled as a Half-Gaussian distribution with variance parameter that is itself exponentially distributed, by integrating over the parameter space, the marginal statistics of the data rate become Laplacian [24], [25].

The disadvantage of using marginal statistics for the bitstream size of each node during each activation interval is the removal of the stochastic dependencies to its transient physical properties 1 . However, in this work we are interested in the expected energy consumption over a time interval and not in the instantaneous variations of energy consumption. Thus, a mean-based analysis using the marginal statistics of the produced bitstream sizes is suitable for this purpose.

\section{B. Energy Consumption Penalties}

Following the push model of the camera node subsystem illustrated in Figure 1 b), each VSN node performs the following operations:

1) Acquisition, processing and transmission: A new frame is acquired by means of a low-power camera sensor and processed with a CPU-intensive algorithm, realized by the multimedia subsystem. Each frame processing (possibly including coding to mitigate channel impairments) produces, on average, $r$ bits for transmission. These bits are pushed to the radio subsystem, which in turn transmits them to the higher tier or, eventually, to the LPBR. Let $a$ Joule $(\mathrm{J})$ be the energy expenditure for acquiring a frame, $g$ be the average energy in Joule (J) required for processing and producing one bit of information to be transmitted and $j$ the average energy required to transmit it to the LPBR or a relay node. Different multimedia applications may incur different

\footnotetext{
${ }^{1}$ e.g. the specifics of what is being monitored at each instant and how the multimedia processing algorithm is operating on the input data
} 
levels of energy consumption for the production of each bit to be transmitted, while the average transmission energy consumption per bit depends only on the specific radio chip used by each wireless sensor node. Hence, the average energy consumed for acquisition, processing and transmission within the active interval of $T$ seconds is $k a+(g+j) \int_{0}^{\infty} \chi_{k} P\left(\chi_{k}\right) d \chi=k a+(g+j) \mathbb{E}\left[\mathcal{X}_{k}\right] \mathbf{J}$, with $\mathbb{E}\left[\mathcal{X}_{k}\right]$ bits comprising the statistical expectation of the data volume corresponding to $k$ frames.

2) Buffering and Idling: As shown in Figure 1, each tier of the sensor network consists of $n$ sensor nodes that communicate with the LPBR (or the relay nodes of the higher tier). The set of all receivers (sink nodes) of each tier has predefined consumption rate of $\frac{s}{T}$ kbps. Under balanced coupling, each sensor node can transmit $\frac{s}{n}$ bits during the analysis time interval of $T$ seconds. We thus identify two cases: if the amount of data generated by the processing phase and relayed from $d$ nodes of the lower tiers is less than $\frac{s}{n}$ bits, the sensor node enters an "idle" state, where $b \mathrm{~J} / \mathrm{bit}$ is consumed for beaconing and other synchronization operations. The energy spent during the idle mode of the analysis time interval is: $b \int_{0}^{\frac{s}{n}}\left(\frac{s}{n}-\chi_{k, d+1}\right) P_{d+1}\left(\chi_{k, d+1}\right) d \chi_{k, d+1}$ $\mathrm{J}$, with $\mathcal{X}_{k, d+1} \sim P_{d+1}\left(\chi_{k, d+1}\right)$ the RV modeling the data rate of a node processing $k$ frames and relaying data from $d$ other independent and identical nodes [with $\mathcal{X}_{k, 1} \equiv \mathcal{X}_{k}$ and $P_{1}\left(\chi_{k}\right) \equiv P\left(\chi_{k}\right)$ ]. Conversely, if the data generated is greater than $\frac{s}{n}$ bits, then the sensor node has to buffer the remaining data in a high-power, typically off-chip, memory. Letting $p$ J be the energy cost of storing one bit of information, the energy spent for buffering during the active time interval is: $p \int_{\frac{s}{n}}^{\infty}\left(\chi_{k, d+1}-\frac{s}{n}\right) P_{d+1}\left(\chi_{k, d+1}\right) d \chi_{k, d+1} \mathrm{~J}$. This case introduces delay, as buffered data will be scheduled for later transmission. Thus, the proposed model is suitable for delay-tolerant multimedia applications [26].

3) Receiving/Buffering and Relaying Data: Under a multitier cluster-tree topology, each node receives $d$ additional data streams from $d$ nodes positioned at the lower tier(s) and relays them along with its own data streams (see Figure 1 for an example with $d=2$ ). Over the analysis interval of $T$ seconds, the energy expenditure corresponding to this process is given by $(h+j) \int_{0}^{\infty} \chi_{k, d} P_{d}\left(\chi_{k, d}\right) d \chi_{k, d}=(h+j) \mathbb{E}\left[\mathcal{X}_{k, d}\right] \mathbf{J}$, with $h \mathrm{~J} /$ bit the average energy required to receive and buffer one bit and $\mathbb{E}\left[\mathcal{X}_{k, d}\right]$ the statistical expectation of the number of bits received from all $d$ nodes of the lower tier(s) during the active time interval. In practice, this energy expenditure is dominated by the receiver power requirements $\mathrm{S}^{2}$ Given that, for IEEE 802.15.4-compliant transceivers, the transceiver power under receive mode is virtually the same regardless if the node is actually receiving data or not, it is irrelevant to the receiver power whether the transmitting node(s) used their entire

\footnotetext{
${ }^{2}$ Energy rates $a, g, j, p, b$ and $h$ may also include fixed, rateindependent costs of the particular multimedia or transceiver hardware (e.g., visual sensor, transceiver or buffer startup and shutdown costs).
}

Table I

NOMENCLATURE TABLE.

\begin{tabular}{|c|c|c|}
\hline Symbol & Unit & Definition \\
\hline$T$ & seconds & Active time interval \\
\hline $\begin{array}{l}n \\
N_{\min } \\
N_{\max }\end{array}$ & - & $\begin{array}{l}\text { Number of transmitting sensor nodes at the } \\
\text { same tier of the cluster-tree topology and } \\
\text { minimum \& maximum nodes allowed by the } \\
\text { application }\end{array}$ \\
\hline $\begin{array}{c}k \\
K_{\min }\end{array}$ & - & $\begin{array}{l}\text { Number of frames captured and processed } \\
\text { within } T \text { seconds and minimum-allowed by the } \\
\text { application }\end{array}$ \\
\hline$r$ & bit & $\begin{array}{l}\text { Average number of bits produced after } \\
\text { processing one frame }\end{array}$ \\
\hline$d$ & - & $\begin{array}{l}\text { Number of additional nodes whose traffic is } \\
\text { relayed by each node at a given tier of the } \\
\text { cluster-tree topology }\end{array}$ \\
\hline$a$ & $\mathrm{~J}$ & $\begin{array}{l}\text { Energy to acquire one frame and initialize the } \\
\text { multimedia processing }\end{array}$ \\
\hline$g$ & $\mathrm{~J} / \mathrm{bit}$ & Energy for processing one bit \\
\hline$j$ & $\mathrm{~J} / \mathrm{bit}$ & Energy for transmitting one bit \\
\hline$p$ & $\mathrm{~J} / \mathrm{bit}$ & $\begin{array}{l}\text { Penalty energy for storing one bit during } \\
\text { receiver overloading }\end{array}$ \\
\hline$b$ & $\mathrm{~J} / \mathrm{bit}$ & $\begin{array}{l}\text { Energy during idle periods for the time interval } \\
\text { corresponding to one bit transmission }\end{array}$ \\
\hline$h$ & $\mathrm{~J} / \mathrm{bit}$ & $\begin{array}{l}\text { Energy for receiving and temporary buffering } \\
\text { one bit under the relay case }\end{array}$ \\
\hline$s$ & bit & $\begin{array}{l}\text { Data volume (bits) of a relay node (or base } \\
\text { station) received within } T \text { seconds }\end{array}$ \\
\hline $\begin{array}{l}\mathcal{X}_{k, d+1} \sim \\
P_{d+1}\left(\chi_{k, d+1}\right)\end{array}$ & bit & $\begin{array}{l}\text { RV modeling the cumulative bits transmitted } \\
\text { by each node, including the bits relayed from } \\
d \text { nodes of lower tiers, after each node } \\
\text { processed } k \text { video frames }\end{array}$ \\
\hline $\mathbb{E}\left[\mathcal{X}_{k, d+1}\right]$ & bit & Statistical expectation of $\mathcal{X}_{k, d+1}$ \\
\hline$E_{c}$ & $\mathrm{~J}$ & $\begin{array}{l}\text { Energy consumption of each individual node } \\
\text { over the analysis time interval } T\end{array}$ \\
\hline$\beta_{\mathrm{D}}, \gamma_{\mathrm{D}}$ & - & $\begin{array}{l}\text { Parameters expressing the combination of the } \\
\text { system energy rates, receiver rate and the mean } \\
\text { of the utilized marginal PDF D for the } \\
\text { solutions obtained along the spatial and } \\
\text { temporal direction }\end{array}$ \\
\hline
\end{tabular}

transmission intervals or not.

\section{Expected Energy Consumption}

Summing all contributions $1 \sim 3$ of the previous subsection, the energy consumption of each node, $E_{\mathrm{c}}$, over the time interval $T$ is:

$$
\begin{gathered}
E_{\mathrm{c}}(n, k)=k a+(g+j) \mathbb{E}\left[\mathcal{X}_{k}\right]+(h+j) \mathbb{E}\left[\mathcal{X}_{k, d}\right] \\
\quad+p \int_{\frac{s}{n}}^{\infty}\left(\chi_{k, d+1}-\frac{s}{n}\right) P_{d+1}\left(\chi_{k, d+1}\right) d \chi_{k, d+1} \\
\quad+b \int_{0}^{\frac{s}{n}}\left(\frac{s}{n}-\chi_{k, d+1}\right) P_{d+1}\left(\chi_{k, d+1}\right) d \chi_{k, d+1} .
\end{gathered}
$$

Adding and subtracting $\quad p \int_{0}^{\frac{s}{n}}\left(\chi_{k, d+1} \quad-\right.$ $\left.\frac{s}{n}\right) P_{d+1}\left(\chi_{k, d+1}\right) d \chi_{k, d+1}$ to (1) leads to: 


$$
\begin{aligned}
E_{\mathrm{c}}(n, k) & =k a+(g+j) \mathbb{E}\left[\mathcal{X}_{k}\right]+(h+j) \mathbb{E}\left[\mathcal{X}_{k, d}\right] \\
& +p \mathbb{E}\left[\mathcal{X}_{k, d+1}\right]-\frac{p s}{n} \\
& +(b+p) \int_{0}^{\frac{s}{n}}\left(\frac{s}{n}-\chi_{k, d+1}\right) P_{d+1}\left(\chi_{k, d+1}\right) d \chi_{k, d+1} .
\end{aligned}
$$

Since the VSN is uniformly formed, all sensors are independent and identical. We can thus establish the relationships:

$$
\begin{gathered}
\forall d>0: \mathbb{E}\left[\mathcal{X}_{k, d+1}\right]=\frac{d+1}{d} \mathbb{E}\left[\mathcal{X}_{k, d}\right], \\
\forall d>0: \mathbb{E}\left[\mathcal{X}_{k, d}\right]=d \mathbb{E}\left[\mathcal{X}_{k}\right],
\end{gathered}
$$

which are based on the fact that the expected number of bits transmitted or received by a node increases linearly with respect to $d$. By modifying (2) based on (3) and (4), we reach:

$$
\begin{aligned}
E_{\mathrm{c}}(n, k) & =k a+[(p+j)(d+1)+h d+g] \mathbb{E}\left[\mathcal{X}_{k}\right]-\frac{p s}{n} \\
& +(b+p) \int_{0}^{\frac{s}{n}}\left(\frac{s}{n}-\chi_{k, d+1}\right) P_{d+1}\left(\chi_{k, d+1}\right) d \chi_{k, d+1} .
\end{aligned}
$$

This equation is the basis for the analytic exploration of the minimum energy consumption under several marginal PDFs characterizing the data production and transmission process.

\section{Analytic Derivation of Minimum EnERgy CONSUMPTION}

Our objective is to derive the spatio-temporal parameters minimizing $E_{\mathrm{c}}(n, k)$ in (5), subject to the spatio-temporal constraints defined in Section III, that is:

$$
\left\{n^{\star}, k^{\star}\right\}=\arg \min _{\forall n, k} E_{\mathrm{c}}(n, k)
$$

with

$$
N_{\text {min }} \leq n \leq N_{\text {max }} \text { and } k \geq K_{\text {min }}
$$

and $\left\{n^{\star}, k^{\star}\right\}$ the values deriving the minimum energy consumption.

In the following, we consider different distributions for $P_{d+1}\left(\chi_{k, d+1}\right)$ and derive the solution for $n$ and $k$ that minimizes the energy consumption, while ensuring the conditions imposed by the application constraints are met. While our analysis is assuming that $n$ and $k$ are continuous variables, once the $\left\{n^{\star}, k^{\star}\right\}$ values are derived, they can be discretized to the points $\left\{\left\lfloor n^{\star}\right\rfloor,\left\lfloor k^{\star}\right\rfloor\right\},\left\{\left\lceil n^{\star}\right\rceil,\left\lceil k^{\star}\right\rceil\right\}$ $\left\{\left\lceil n^{\star}\right\rceil,\left\lfloor k^{\star}\right\rfloor\right\}\left\{\left\lfloor n^{\star}\right\rfloor,\left\lceil k^{\star}\right\rceil\right\}$ [if all four satisfy the constraints of [7] in order to check which discrete pair of values derives the minimum energy consumption in (5). This is because: $(i)$ the energy functions under consideration are continuous and differentiable; and (ii) we shall show that a unique minimum is found for (5) that is parametric to the setting of the temporal constraint $\left(K_{\min }\right)$. As such, the analysis on the continuous variable space can be directly mapped onto the discrete variable set under the aforementioned discretization.

\section{A. Definitions of Data Transmission PDFs under Consid- eration and Infeasibility of Global Minimum of $E_{c}(n, k)$}

When one has limited or no knowledge about the cumulative data transmitted by each VSN node during the active time interval, one can assume that $P_{d+1}\left(\chi_{k, d+1}\right)$ is uniform over the interval $[0,2 k r(d+1)]$.

Definition 1. $\left(P_{d+1}\left(\chi_{k, d+1}\right)\right.$ is Uniform $)$ : We define $P_{d+1}\left(\chi_{k, d+1}\right)$ as the Uniform distribution when:

$$
P_{d+1}\left(\chi_{k, d+1}\right)= \begin{cases}\frac{1}{2 k r(d+1)} & 0 \leq \chi_{k, d+1} \leq 2 k r(d+1) \\ 0 & \text { otherwise }\end{cases}
$$

with $\mathbb{E}_{\mathrm{U}}\left[\mathcal{X}_{k}\right]=k r$ [and $\left.\mathbb{E}_{\mathrm{U}}\left[\mathcal{X}_{k, d+1}\right]=k r(d+1)\right]$ corresponding to the mean value of the data transmitted by a node that produces $k$ frames of $r$ bits each on average (and relays information from $d$ other nodes).

Corollary 1. When $P_{d+1}\left(\chi_{k, d+1}\right)$ is Uniform, there exists no global solution to (6) in its unconstrained form.

Proof: Using (8) in (5) leads to:

$$
\begin{aligned}
E_{\mathrm{c}, \mathrm{U}}(n, k) & =k[a+r[(p+j)(d+1)+h d+g]] \\
& -\frac{p s}{n}+\frac{s^{2}(b+p)}{4 n^{2} k r(d+1)} .
\end{aligned}
$$

To obtain the solution to (6) under the energy consumption given by (9), one can search for critical points of $E_{\mathrm{c}, \mathrm{U}}$. By definition, a critical point of a multidimensional function is the point where the gradient of the function is equal to zero. Imposing that the derivatives of $E_{\mathrm{c}, \mathrm{U}}$ with respect to $n$ and $k$ are both equal to zero leads to:

$$
\left\{\begin{aligned}
\frac{\partial E_{\mathrm{c}, \mathrm{U}}}{\partial n} & =\frac{p s}{n^{2}}-\frac{s^{2}(b+p)}{2 n^{3} k r(d+1)}=0 \\
\frac{\partial E_{\mathrm{c}, \mathrm{U}}}{\partial k} & =a+r[(p+j)(d+1)+h d+g] \\
& -\frac{s^{2}(b+p)}{4 n^{2} k^{2} r(d+1)}=0
\end{aligned}\right.
$$

Solving $\frac{\partial E_{\mathrm{c}, \mathrm{U}}}{\partial n}=0$ for $n$ gives $n=\frac{s(b+p)}{2 k r p(d+1)}$. Substituting this solution in $\frac{\partial E_{\mathrm{c}, \mathrm{U}}}{\partial k}=0$ and solving for $a$, leads to $a<0$. However, this is not feasible since $a$ is the energy cost to acquire one frame. Hence, under the physical constraints of the problem, there is no single (global) solution $\left\{n^{\star}, k^{\star}\right\} \in \mathbb{R} \times \mathbb{R}$ to (6) in its unconstrained form, i.e. when one ignores the constraints of (7).

We now extend the analysis towards other PDFs for the data transmission, which are frequently encountered in practice.

Definition 2. $\left(P_{d+1}\left(\chi_{k, d+1}\right)\right.$ is Pareto $)$ : We consider $P_{d+1}\left(\chi_{k, d+1}\right)$ as the Pareto distribution with scale $v$ and shape $\alpha>1$ when:

$$
P_{d+1}\left(\chi_{k, d+1}\right)=\left\{\begin{array}{ll}
\alpha \frac{v^{\alpha}}{\chi_{k, d+1}^{\alpha+1}}, & \chi_{k, d+1} \geq v \\
0, & \text { otherwise }
\end{array} .\right.
$$


Setting $v=\frac{\alpha-1}{\alpha} k r(d+1)$ leads to $\mathbb{E}_{\mathrm{P}}\left[\mathcal{X}_{k}\right]=k r$, i.e. we match the expected data volume to that of the Uniform $P D F$.

The Pareto distribution has been used, amongst others, to model the marginal data size distribution of TCP sessions that contain substantial number of small files and a few very large ones [27], [28]. It has also been used to model multimedia traffic packet sizes in several works, e.g. by Kumar [29].

Definition 3. $\left(P_{d+1}\left(\chi_{k, d+1}\right)\right.$ is Exponential): We consider $P_{d+1}\left(\chi_{k, d+1}\right)$ as the Exponential distribution when:

$$
P_{d+1}\left(\chi_{k, d+1}\right)=\frac{1}{k r(d+1)} \exp \left(-\frac{1}{k r(d+1)} \chi_{k, d+1}\right) .
$$

with $\mathbb{E}_{\mathrm{E}}\left[\mathcal{X}_{k}\right]=k r$ [and $\left.\mathbb{E}_{\mathrm{E}}\left[\mathcal{X}_{k, d+1}\right]=k r(d+1)\right]$ corresponding to the mean value of the data transmitted by a node that produces $k$ frames of $r$ bits each on average (and relays information from $d$ other nodes).

We remark that the marginal statistics of MPEG video traffic have often been modeled as exponentially decaying [30].

We conclude by considering $P_{d+1}\left(\chi_{k, d+1}\right)$ as the HalfGaussian distribution with mean $\mathbb{E}_{\mathrm{H}}\left[\mathcal{X}_{k}\right]=k r$. This distribution has been widely used in data gathering problems in science and engineering when the modeled data has non-negativity constraints. Some recent examples include the statistical characterization of motion vector data sizes in Wyner-Ziv video coding algorithms suitable for VSNs [31], or the statistical characterization of sample amplitudes captured by an image sensor [24], [25], [32].

Definition 4. $\left(P_{d+1}\left(\chi_{k, d+1}\right)\right.$ is Half-Gaussian): We consider $P_{d+1}\left(\chi_{k, d+1}\right)$ as the Half-Gaussian distribution when:

$P_{d+1}\left(\chi_{k, d+1}\right)= \begin{cases}\frac{2}{\pi k r(d+1)} \exp \left(-\frac{\chi_{k, d+1}^{2}}{\pi k^{2} r^{2}(d+1)^{2}}\right), & \chi_{k, d+1} \geq 0 \\ 0, & \chi_{k, d+1}<0\end{cases}$

with $\mathbb{E}_{\mathrm{H}}\left[\mathcal{X}_{k}\right]=k r$ [and $\mathbb{E}_{\mathrm{H}}\left[\mathcal{X}_{k, d+1}\right]=k r(d+1)$ ] corresponding to the mean value of the data transmitted by a node that produces $k$ frames of $r$ bits each on average (and relays information from $d$ other nodes).

Corollary 2. When $P_{d+1}\left(\chi_{k, d+1}\right)$ is the Pareto, Exponential or Half-Gaussian distribution, given by (11)-(13), there exists no global solution to (6) in its unconstrained form. comes:

Proof: Under (11), the energy expression of (5) be-

$$
\begin{aligned}
E_{\mathrm{c}, \mathrm{P}} & =k[a+r[(p+j)(d+1)+h d+g]] \\
& +\frac{b s}{n}+(b+p)\left(\frac{v^{\alpha} n^{\alpha-1}}{s^{\alpha-1}(\alpha-1)}-\frac{\alpha v}{\alpha-1}\right) .
\end{aligned}
$$

In addition, replacing (12) in the energy expression of
(5), we obtain:

$$
\begin{aligned}
E_{\mathrm{c}, \mathrm{E}} & =k[a+r[(p+j)(d+1)+h d+g]]+\frac{b s}{n} \\
& +(b+p)\left[k r(d+1)\left(\exp \left(-\frac{s}{n k r(d+1)}\right)-1\right)\right] .
\end{aligned}
$$

Finally, replacing (13) in the energy expression of (5), we obtain:

$$
\begin{gathered}
E_{\mathrm{c}, \mathrm{H}}=k[a+[r(p+j)(d+1)+h d+g]]-\frac{p s}{n}+(b+p) \\
\times\left[k r(d+1)\left(\exp \left(-\frac{s^{2}}{\pi k^{2} r^{2} n^{2}(d+1)^{2}}\right)-1\right)\right. \\
\left.+\frac{s}{n} \operatorname{erf}\left(\frac{s}{\sqrt{\pi} k r n(d+1)}\right)\right] .
\end{gathered}
$$

To obtain the solution to (6) under the energy consumption given by (9), one can search for critical points of $E_{\mathrm{c}, \mathrm{P}}, E_{\mathrm{c}, \mathrm{E}}$ and $E_{\mathrm{c}, \mathrm{H}}$. Similarly as for Corollary 1 , it is straightforward to show that imposing that the derivatives of $E_{\mathrm{c}, \mathrm{P}}, E_{\mathrm{c}, \mathrm{E}}$ and $E_{\mathrm{c}, \mathrm{H}}$ with respect to $n$ and $k$ are both equal to zero leads to solutions that require $a<0$ (detailed derivations omitted), which is not physically feasible since $a$ is the energy cost to acquire one frame.

It follows from Corollary 1 and 2 that, under the physical constraints of the problem, there is no single (global) solution $\left\{n^{\star}, k^{\star}\right\} \in \mathbb{R} \times \mathbb{R}$ to (6) in its unconstrained form, i.e., when one ignores the constraints of (7). However, we may consider each dimension individually (i.e., perform univariate minimization along the $n$ or $k$ dimension) in order to find a local or global minimum for that particular dimension and then choose for the other dimension the value that minimizes (6) under the spatio-temporal constraints of (7). Subsequently, we can identify if the derived minima are unique under the imposed constraints and whether the entire region of support of the energy function under these constraints has been covered by the derived solutions. Following this approach, the main results are presented in the following subsection. The detailed derivations are contained in the Appendices.

\section{B. Main Results: Parametric Minima of $E_{c}(n, k)$}

Proposition 1. When the data transmitted by each VSN node follows the Uniform, Pareto or Exponential distributions of Definitions 1-3, the sets of solutions giving the minimum energy consumption in (6) under the spatiotemporal constraints of (7) are:

$$
\left\{n^{\star}, k^{\star}\right\}_{\mathrm{D}}= \begin{cases}\left(N_{\max }, \frac{\gamma_{\mathrm{D}}}{N_{\max }}\right) & \text { if } K_{\min } \leq \frac{\gamma_{\mathrm{D}}}{N_{\max }} \\ \left(N_{\max }, K_{\min }\right) & \text { if } \frac{\gamma_{\mathrm{D}}}{N_{\max }}<K_{\min }<\frac{\beta_{\mathrm{D}}}{N_{\max }} \\ \left(\frac{\beta_{\mathrm{D}}}{K_{\min }}, K_{\min }\right) & \text { if } \frac{\beta_{\mathrm{D}}}{N_{\max }} \leq K_{\min } \leq \frac{\beta_{\mathrm{D}}}{N_{\min }} \\ \left(N_{\min }, K_{\min }\right) & \text { if } K_{\min }>\frac{\beta_{\mathrm{D}}}{N_{\min }}\end{cases}
$$

with $\mathrm{D} \in\{\mathrm{U}, \mathrm{P}, \mathrm{E}\}$ indicating each of the three distributions, and $\beta_{\mathrm{D}}$ and $\gamma_{\mathrm{D}}$ defined by:

$$
\beta_{\mathrm{U}}=\frac{s(b+p)}{2 p r(d+1)},
$$




$$
\begin{gathered}
\gamma_{\mathrm{U}}=\frac{s}{2} \sqrt{\frac{b+p}{r(d+1)[a+r[(p+j)(d+1)+h d+g]]}} \\
\gamma_{\mathrm{P}}=\frac{s \alpha}{r(\alpha-1)(d+1)}\left(\frac{r[(b-j)(d+1)-h d-g]-a}{r(d+1)(b+p)}\right)^{\frac{1}{\alpha-1}} \\
\beta_{\mathrm{P}}=\frac{s \alpha}{r(\alpha-1)(d+1)}\left(\frac{b}{b+p}\right)^{\frac{1}{\alpha}} \\
\beta_{\mathrm{E}}=\frac{s}{r(d+1) \ln \left(\frac{b+p}{p}\right)}
\end{gathered}
$$

and

$$
\gamma_{\mathrm{E}}=-\frac{s}{r(d+1)\left[W\left(-\frac{1}{\exp } \frac{-a+r[(b-j)(d+1)-h d-g]}{r(d+1)(b+p)}\right)+1\right]},
$$

with $W(\cdot)$ the Lambert product-log function [33]. For the particular case when $\mathrm{D}=\mathrm{E}$ (Exponential PDF), (17) holds under the condition that $p>b$, i.e., the penalty energy to buffer bits is higher than beaconing energy.

Proof: See Appendix A

Proposition 2. When the data transmitted by each VSN node follows the Half-Gaussian distribution of Definition 4, the set of solutions giving the minimum energy consumption in (6) under the spatio-temporal constraints of (7) is:

$$
\left\{n^{\star}, k^{\star}\right\}_{\mathrm{H}}= \begin{cases}\left(N_{\max }, K_{\min }\right) & \text { if } K_{\min } \leq \frac{\beta_{\mathrm{H}}}{N_{\max }} \\ \left(\frac{\beta_{\mathrm{H}}}{K_{\min }}, K_{\min }\right) & \text { if } \frac{\beta_{\mathrm{H}}}{N_{\max }}<K_{\min } \leq \frac{\beta_{\mathrm{H}}}{N_{\min }} \\ \left(N_{\min }, K_{\min }\right) & \text { if } K_{\min }>\frac{\beta_{\mathrm{H}}}{N_{\min }}\end{cases}
$$

with

$$
\beta_{\mathrm{H}}=\frac{s}{\sqrt{\pi} r(d+1) \operatorname{erf}^{-1}\left(\frac{p}{b+p}\right)} .
$$

Proof: The proof follows the same steps as for the previous cases and it is summarized in Appendix A-D.

\section{Discussion}

The key observation from Propositions 1 and 2 is that, regardless to the distribution used for modeling the data production process, the solutions giving the minimum energy consumption attain the same mathematical form. Specifically, when the initial constraint on the minimum number of frames captured and processed within $T$ seconds, $K_{\min }$, is higher than the threshold value: $\frac{\beta_{\mathrm{D}}}{N_{\min }}$, the optimal solution is the one where $N_{\min }$ nodes process $K_{\min }$ frames each (i.e., the minimum setting possible for nodes and frames-per-node). If $K_{\min }$ is smaller or equal than this threshold, therefore facilitating more nodes within each tier of the VSN, the optimal number of nodes, $n^{\star}$, derived by Propositions 1 and 2 , increases to $\frac{\beta_{\mathrm{D}}}{K_{\min }}$. However, when $n^{\star}$ reaches the constraint on the maximum number of nodes, $N_{\text {max }}$, then the optimal solution for each node is to use a frame setting that is higher than $K_{\min }$. The latter is true for Proposition 1; however, for Proposition 2 (HalfGaussian PDF), the corresponding optimal frame setting was found to be imaginary regardless to the specific system parameter. Therefore, the optimal solution for this case is always $k^{\star}=K_{\min }$.

In terms of relevance to practical applications, the results of this section can be used to assess the impact of the spatio-temporal constraints and the data production and transmission process (as characterized by its marginal PDF) on the energy consumption of VSNs, under a variety of energy consumption rates for the radio and multimedia subsystems. For example, under given energy availability from the node battery and predetermined system activation time $(T)$, this allows for the determination of appropriate hardware to be used (i.e. $j, h, b, p, a$ and $g$ parameters) in order to meet the spatio-temporal constraints of the application. Moreover, via the analysis of the previous four subsections, one can optimize the system under the assumption of a certain marginal PDF characterizing the data production and transmission process of each node.

Conversely, under particular technology (i.e. given $j, h$, $b, p, a$ and $g$ parameters) and given configuration for the VSN in terms of number of nodes and frames to capture within the activation time interval, one can determine the required energy in order to achieve the designated visual data gathering task. Furthermore, under the proposed framework, one can determine the data production and transmission (marginal) PDFs that meet predetermined energy supply and spatio-temporal constraints.

Although we do not claim that the utilized PDFs cover all possible scenarios that can be encountered in practice, they comprise an ensemble of distributions that includes several important aspects, i.e.,: (i) the maximum-entropy PDF (Uniform); (ii) well-known distributions characterizing the transmission rate of real-world systems (Exponential and Half-Gaussian) [24], [25], [30]-[32], and (iii) a parameterized distribution (Pareto) that corresponds to the continuous equivalent to Zipf's law for generalized frequency of occurrences of physical phenomena; moreover, if $\alpha=k r$, the Pareto distribution corresponds to near fixedrate transmission with rate $k r$. Beyond the cases considered in this paper, if another distribution provides a better fit to a particular deployment, the steps of Propositions 1 and 2 can be used to provide a characterization of the available solution space. Moreover, given that the results of Propositions 1 and 2 are applicable per node, if the considered scenario involves a non uniformly-formed VSN, the same analysis applies for each node of each cluster-tree tier, albeit with the use of:

1) a different PDF per sensor, leading to a mixture of PDFs for the relayed traffic, with the resulting distribution being the convolution of the intermediate distributions;

2) unbalanced coupling in (2) and (3), i.e., the $i$ th node transmitting $s_{i}$ bits during the analysis time interval of $T$ seconds, with $s_{i}$ allocated by the utilized protocol during the cluster formation [18], [22], [26], [34];

3 ) the $i$ th node of each cluster relaying traffic from $d_{i}$ nodes, and, in general, $d_{i} \neq d_{i^{\prime}}$ for $i \neq i^{\prime}$.

Given that a numerical package (e.g., Mathematica or Matlab Symbolic) can be used for the calculation of: (i) the con- 
volution of $d_{i}+1$ distributions $P_{d_{i}+1}\left(\chi_{k, d_{i}+1}\right)$ (corresponding to the mixture of $d_{i}+1$ PDFs of the $i$ th node of each tier) and (ii) the $\int_{0}^{s_{i}}\left(s_{i}-\chi_{k, d_{i}+1}\right) P_{d_{i}+1}\left(\chi_{k, d_{i}+1}\right) d \chi_{k, d_{i}+1}$ term of (2), we do not expand on these cases further.

Overall, our proposed energy consumption model and the associated analytic results can be used in many ways for early-stage exploration of system, network, and data production parameters in VSNs that match the design specifications of classes of application domains. Such application examples are given in Section $\mathrm{V}$.

\section{Evaluation of the Analytic Results}

To validate the proposed analytic model of (5) and Propositions 1 and 2 for the settings leading to the minimum energy consumption, we performed a series of experiments based on a visual sensor network matching the system model of Section $\Pi$ and an energy-measurement testbed. Specifically, each visual node of the sensor network is composed of a BeagleBone Linux Computer (multimedia subsystem) attached to a TelosB sensor node for low-power wireless communications (radio subsystem) [12]. Each BeagleBone is equipped with a RadiumBoard CameraCape to provide for the video frame acquisition. For energy-efficient processing, we downsampled all input images to QVGA (320x240) resolution.

In order to measure the energy consumption of each VSN node, we captured the real-time current consumption at two high-tolerance $1 \mathrm{Ohm}$ resistors, the first of which was placed in series with the multimedia and the second in series with the radio subsystem of each visual node. A Tektronix MDO4104-6 oscilloscope was used for the two current consumption captures of each experiment. Further, our deployment involved: $(i)$ a TelosB node serving as the LPBR and collecting all bitstreams and 2 to 32 visual nodes positioned within four adjacent rooms and the corridor of the same floor of the Department of Electrical and Electronic Engineering at University College London [following the layout of Figure 11.a)]; (ii) a uniformly-formed hierarchical cluster-tree network topology with $n=2$ to $n=16$ nodes per network tier and the recently-proposed (and available as open source) TFDMA protocol [7] for contention-free MAC-layer coordination; (iii) no WiFi or other IEEE802.15.4 networks concurrently operating in the utilized channels of the $2.4 \mathrm{GHz}$ band. Even if IEEE802.11 or other IEEE802.15.4 networks coexist with the proposed deployment, well-known channel hopping schemes like $\mathrm{TSCH}$ [35] or interference-avoidance schemes [36] can be used at the MAC layer to mitigate such external interference while maintaining a balanced cluster tree topology in the WSN.

TFDMA ensures collision-free multichannel communications with guaranteed timeslots via a fair time-division multiple access (TDMA) schedule constructed within each of the utilized channels of the IEEE802.15.4 physical layer via beacon packet exchanges [7]. Protocols such as TFDMA, the TSCH mode of IEEE 802.15.4e-2012 [3] and other balanced cluster-tree-based MAC-layer protocols [2],
[8], [22], [34], allow for collision-free, uniformly-formed, cluster-tree based VSNs to be formed via the combination of fair TDMA scheduling and channel allocation or channel hopping. Experiments have shown that such protocols can scale to hundreds or even thousands of nodes [37]. Therefore, our evaluation is pertinent to such scenarios that may be deployed in the next few years within the IoT paradigm [4], [5].

\section{A. Radio Subsystem}

For what concerns the radio subsystem, each TelosB runs the low-power Contiki 2.6 operating system. Given that the utilized TFDMA protocol ensures collision-free transmissions from each node, we enabled the low-power NullMAC and NullRDC options of the Contiki OS that disable the default MAC queuing and backoff mechanisms. This led to data consumption rate at the application layer of $\frac{s}{T}=144 \mathrm{kbps}$.

Given that varying the transmission power level has minimal effect on the VSN node energy consumption (since most of the transceiver current consumption is due to reception) and may compromise error-free data reception, we utilized the maximum transmit power, which led to reliable data transmission under the collision-free timeslot allocation of TFDMA. Under these operational settings, the average transmission cost per bit of information, $j \mathrm{~J} / \mathrm{bit}$, as well as the cost for beaconing, $b \mathrm{~J} / \mathrm{bit}$, and buffering, $p \mathrm{~J} / \mathrm{bit}$, were established experimentally by repeating several dedicated energy-measurement tests with the TelosB subsystem; their values are shown at the top half of Table II and we have experimentally verified that they remained constant over several activation intervals.

\section{B. Multimedia Subsystem}

Since the energy consumption of the multimedia subsystem is application-dependent, we focused on two different applications, namely: (i) encoding and transmission of JPEG video frames and (ii) extraction and transmission of local features for visual analysis. These two scenarios represent a wide range of practical VSN-related deployments proposed recently [1], [10], [14], [17], [38]-[41].

1) Differential Motion JPEG (MJPEG) encoding: We used a hybrid DCT-DPCM encoder, such as the one presented in [39]. In this system, the first frame of the video sequence is JPEG encoded and transmitted. For the subsequent frames, only the difference between two adjacent frames is encoded. The encoding process follows the standard JPEG baseline, i.e., quantization of the Discrete Cosine Transform (DCT) coefficients followed by run length coding (RLE) and Huffman coding.

2) Visual Features extraction: Several visual analysis tasks can be performed by disregarding the pixel representation of an image, and relying only on a much more compact representation based on local visual features [16]. In a nutshell, salient keypoints of an image are identified by means of a detector, and a descriptor is computed from the pixel values belonging to the image 
Table II

VISUAL SENSOR ENERGY AND BITRATE PARAMETERS.

\begin{tabular}{|c|c|c|c|}
\hline Parameter & Description & Unit & Value \\
\hline \hline \multicolumn{4}{|c|}{ Radio Subsystem (TelosB) } \\
\hline \hline$\frac{s}{T}$ & Data consumption rate & kbps & 144 \\
\hline$j$ & Transmission cost & $\mathrm{J} / \mathrm{bit}$ & $2.20 \times 10^{-7}$ \\
\hline$h$ & Receiving cost & $\mathrm{J} / \mathrm{bit}$ & $2.92 \times 10^{-6}$ \\
\hline$b$ & Beaconing/idling cost & $\mathrm{J} / \mathrm{bit}$ & $1.90 \times 10^{-7}$ \\
\hline$p$ & Buffering cost & $\mathrm{J} /$ bit & $2.86 \times 10^{-7}$ \\
\hline \hline \multicolumn{4}{|c|}{ Multimedia Subsystem (BeagleBone) } \\
\hline \hline$a_{\mathrm{ACQ}}$ & Acquisition cost & $\mathrm{J}$ & $5.00 \times 10^{-3}$ \\
\hline$a_{\mathrm{JPE}}$ & Initialization cost (JPEG) & $\mathrm{J}$ & $1.40 \times 10^{-2}$ \\
\hline$a_{\mathrm{VF}}$ & Initialization cost (Visual Feat.) & $\mathrm{J}$ & $7.79 \times 10^{-3}$ \\
\hline$g_{\mathrm{JPEG}}$ & Processing cost (JPEG) & $\mathrm{J} /$ bit & $4.40 \times 10^{-8}$ \\
\hline$g_{\mathrm{VF}}$ & Processing cost (Visual Feat.) & $\mathrm{J} / \mathrm{bit}$ & $1.90 \times 10^{-8}$ \\
\hline \multicolumn{4}{|c|}{}
\end{tabular}

patch around each keypoint. Here, we focus on cornerlike local features produced by processing each frame of the input video sequence with the FAST corner detector [42], which is optimized for fast extraction of visual features on low-power devices. Each detected keypoint is then described by means of a binary descriptor: we used the BRIEF algorithm [43], which outputs descriptors of 64 bytes each.

Dedicated energy-measurement tests were performed with the Beaglebone multimedia subsystem by varying the encoding quality factor for differential MJPEG, while for features extraction, we varied the FAST detection threshold. This allowed us to trace curves in the energy-rate plane and to obtain the average energy cost per bit, as well as the average initialization cost per frame for both the application scenarios, which are reported at the bottom half of Table III. The cost of acquiring one frame was derived from the specifications of the AptinaMT9M114 image sensor mounted on the CameraCape and is reported in Table II The overall acquisition cost for one frame is established as $a=a_{\mathrm{ACQ}}+a_{\mathrm{JPEG}}$ for the JPEG case and $a=a_{\mathrm{ACQ}}+a_{\mathrm{VF}}$ for the visual-feature extraction case.

\section{Model Validation via Monte-Carlo-generated Data}

Under the settings described previously and shown in Table III, our first goal is to validate the analytic expressions of Section [III that form the mathematical foundation for Propositions 1 and 2, namely (9), (14), (15) and (16). To this end, we create a controlled multimedia data production process on each VSN node by: (i) artificially creating several sets of bitstream sizes according to the marginal PDFs of Section III] via rejection sampling [44]; (ii) setting the mean data size per video frame to $r=5.2 \mathrm{kbit}$; (iii) setting $d=0$ (no relaying) and $d=2$ for each distribution. The sets containing data sizes are copied onto the readonly memory of each sensor node during deployment. At run time, each node fetches a new frame size from the preloaded set, produces artificial data according to it (akin to receiving the information from the multimedia subsystem) and transmits the information to the LPBR following the process described in the system model of Section III Depending on the frame size, the node can enter in idling/beaconing state, or it can buffer the data exceeding the allocated TFDMA slots. This controlled experiment with Monte-Carlo-generated datasets creates the conditions that match our statistical characterization and can therefore confirm the validity of our derivations.

We report here energy measurements obtained under varying values of $n$ and $k$. The chosen active time interval was set to be $T=154$ seconds and, beyond measuring the accuracy of the model versus experiments, we also compared the theoretically-optimal values for $k$ and $n$ according to Section IIII with the ones producing the minimum energy consumption in the experiments. For the reported experiments of Figures 2 and Table III, the spatiotemporal constraints were: $N_{\min }=2, N_{\max }=16$ and $K_{\text {min }}=2 T$ frames, i.e. two frames per second. All our reported measurements and the values for $k$ are normalized to a one-second interval for easier interpretation of the results.

As one can see from Figures 22, and Table [III the theoretical results match the experimental results for all the tested distributions, with the maximum percentile error between them limited to $6.34 \%$ and all the coefficients of determination $R^{2}$ between the experimental and the model points being above 0.995 . In addition, the theoreticallyobtained optimal values for $\left\{n^{\star}, k^{\star}\right\}$ from (17) and (24) are always in agreement with the experimentally-derived values that were found to offer the minimum energy consumption under the chosen spatio-temporal constraints. We have observed the same level of accuracy for the proposed model under a variety of data sizes $(r)$, active time interval durations $(T)$, number of relay nodes $(d)$ and spatiotemporal constraints $\left(N_{\min }, N_{\max }\right.$ and $\left.K_{\min }\right)$, but omit these repetitive experiments for brevity of exposition.

As mentioned in Section $\mathrm{III}_{-} \mathrm{C}$, the optimal solution does not always correspond to the minimum allowable number of frames (i.e., $K_{\min }$ ). For instance, Figure 3 shows the theoretical and experimental results obtained by setting $N_{\min }=2, N_{\max }=6$ and $K_{\min }=\frac{T}{2}$ (i.e., one frame every two seconds), and using the Uniform distribution. Under these settings, the optimal solution was found to be $\left\langle n^{\star}=6, k^{\star}=T\right\rangle$, thereby confirming the validity of the proposed model.

\section{Applications}

In order to assess the proposed model against real application data, we repeated the experimental measurements described in Section IV-C for both application scenarios and under the same spatio-temporal constraints $\left(N_{\min }=2\right.$, $N_{\max }=16, K_{\min }=2 T$, i.e. two frames per second), this time capturing and processing real data from our deployment and utilizing the energy parameters of Table II for the proposed analytic model. We then matched ${ }^{3}$ the energy measurements with one of the energy functions derived in Section III Specifically, we found that the results

\footnotetext{
${ }^{3}$ Fitting is performed by matching the average data size $r$ of each distribution to the average data size of the JPEG compressed frames or the set of visual features.
} 


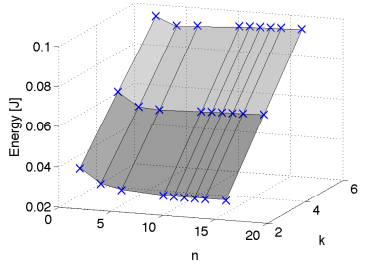

(a) Uniform PDF $(d=0)$

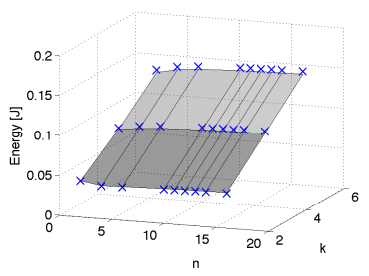

(e) Uniform PDF $(d=2)$

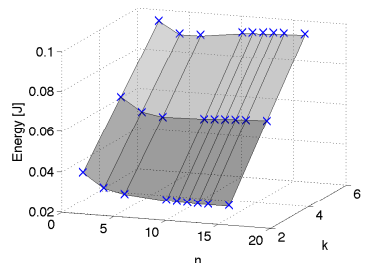

(b) Pareto PDF ( $\alpha=4, d=0)$

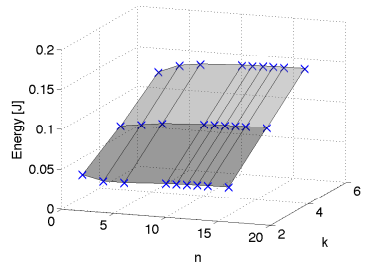

(f) Pareto PDF ( $\alpha=4, d=2)$

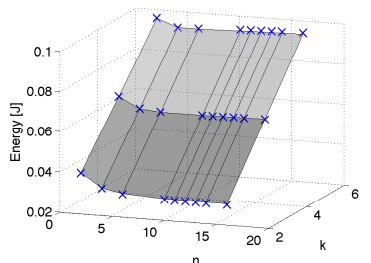

(c) Exponential PDF $(d=0)$

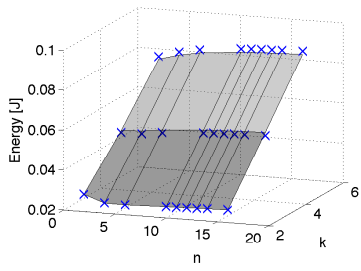

(g) Exponential PDF $(d=2)$

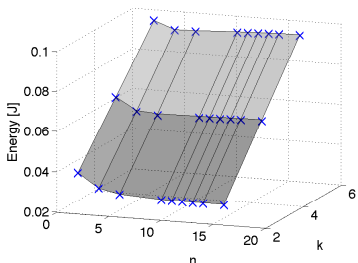

(d) Half-Gaussian PDF $(d=0)$

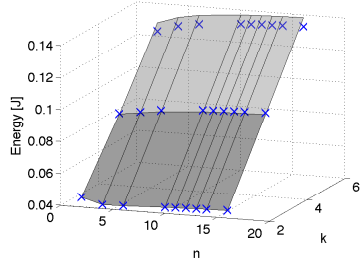

(h) Half-Gaussian PDF $(d=2)$

Figure 2. Each column shows the results corresponding to a marginal PDF characterizing the data transmission process. The grayscale surfaces show the energy consumption of a single camera sensor node in function of the number of frames per second and the total number of nodes. The blue crosses correspond to the value of the consumed energy as measured from the sensor network testbed. All energy values and frames $(k)$ are normalized to an one-second interval.

Table III

DiFFERENCES BETWEEN THE THEORETICAL AND EXPERIMENTAL RESULTS AND THE OPTIMAL VALUES, $\left\{n^{\star}, k^{\star}\right\}_{\text {D }}$, FOR THE NUMBER OF NODES AND THE FRAMES-PER-SECOND FOR THE CONSIDERED DATA TRANSMISSION (MARGINAL) PDFS UNDER THE SETTINGS OF FIGURE 2 AND $d=2$ (EACH NODE RELAYING DATA FROM TWO OTHER NODES).

\begin{tabular}{|c|c|c|c|c|c|c|c|c|}
\hline & \multicolumn{4}{|c|}{$d=0$ (no relay transmission) } & \multicolumn{3}{c|}{$d=2$ (relaying from two other nodes) } \\
\hline $\begin{array}{c}\text { Transmission } \\
\text { PDF }\end{array}$ & $\begin{array}{c}\text { Mean } \\
\text { error (\%) }\end{array}$ & $\begin{array}{c}\text { Max. } \\
\text { error } \mathbf{( \% )}\end{array}$ & $\begin{array}{c}R^{2} \\
\text { coeff. }\end{array}$ & $\begin{array}{c}\text { Theoretical } \\
\text { optimum }\end{array}$ & $\begin{array}{c}\text { Mean } \\
\text { error (\%) }\end{array}$ & $\begin{array}{c}\text { Max. } \\
\text { error (\%) }\end{array}$ & $\begin{array}{c}R^{2} \\
\text { coeff. }\end{array}$ & $\begin{array}{c}\text { Theoretical } \\
\text { optimum }\end{array}$ \\
\hline \hline Uniform & 1.19 & 2.24 & 0.9982 & $\{12,2\}$ & 1.37 & 2.21 & 0.9921 & $\{4,2\}$ \\
\hline Pareto $(\alpha=4)$ & 1.40 & 3.6 & 0.9980 & $\{16,2\}$ & 1.51 & 6.34 & 0.99983 & $\{6,2\}$ \\
\hline Exponential & 1.36 & 2.85 & 0.9984 & $\{15,2\}$ & 3.05 & 4.52 & 0.9895 & $\{5,2\}$ \\
\hline Half-Gaussian & 0.37 & 0.69 & 0.9991 & $\{13,2\}$ & 1.33 & 2.24 & 0.9977 & $\{4,2\}$ \\
\hline
\end{tabular}

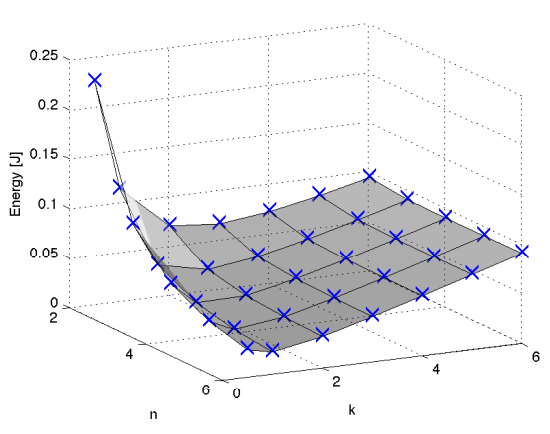

Figure 3. Predicted (gray surface) and measured (blue crosses) energy consumption of a single camera node in function of the number of frames per second and the total number of nodes, for the case of Uniform distribution under $N_{\min }=2, N_{\max }=6$ and $K_{\min }=\frac{T}{2}$. All energy values and frames $(k)$ are normalized to an one-second interval.

matched best the Pareto distribution with parameters $\alpha=4$, $v=k r$ and $r=20.6 \mathrm{kbit}$ for the JPEG case and $r=11.7$ kbit for the visual features case, as shown in Figures 4(a) and 4(b), with coefficient of determination value $R^{2} \cong 0.97$ for the JPEG case and $R^{2} \cong 0.96$ for the visual features case. Similarly as before, all reported energy values and number of frames are normalized to a one-second interval for easier interpretation of the results.
Given the high accuracy of the Pareto-based energy model against the application results, we utilized the settings for the minimum energy consumption derived for the Pareto case [see [17] ] to ascertain the energy saving that can be potentially achieved against arbitrary (ad-hoc) settings. As an example, in Tables IV and $\mathrm{V}$, we consider two different cases for each application scenario, characterized by different spatio-temporal constraints. For each case, we compare the optimal solution given by 17) (for the Pareto case) with an ad-hoc "least-cost" solution that assumes values equal to the minimum spatio-temporal constraints (under the intuitive assumption that less nodes and less frames-per-second lead to smaller energy consumption). Evidently, the proposed approach allows for $8 \%$ to $37 \%$ energy savings in comparison to the ad-hoc settings in both applications under consideration. As such, its usage can be envisaged for early-stage testing of plausible application deployments with respect to their energy efficiency in order to determine the impact of various options for the multimedia and radio subsystems, as well as the best spatiotemporal parameters to consider, prior to more detailed experimentation in the field. 


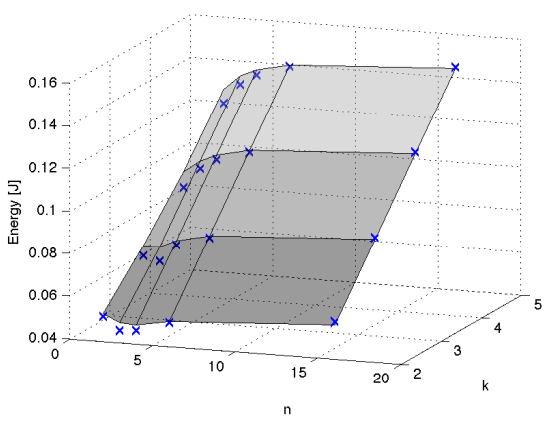

(a) DCT-DPCM coding, $R^{2}=0.9698$

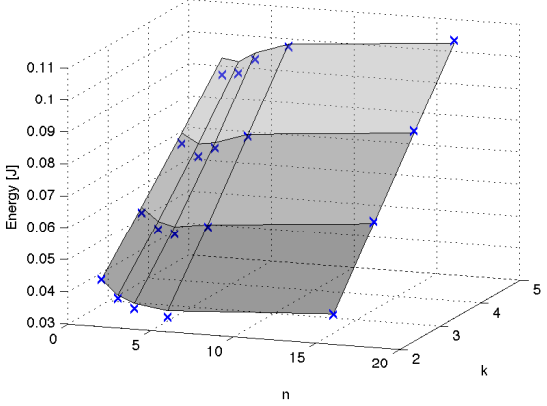

(b) Visual features extraction, $R^{2}=0.9596$

Figure 4. The energy function for the two considered application scenarios. The grayscale surfaces represent the fitted energy function obtained with the Pareto PDF, while the blue crosses represent the experimental measurements. All energy values and frames $(k)$ are normalized to a one-second interval.

Table IV

MOTION JPEG APPLICATION SCENARIO

\begin{tabular}{|c||c|c|c|}
\hline Constraints & Ad-hoc deployment & Proposed approach & Gain \\
\hline \hline$K_{\min }=0.7$ & $k=0.7$ & $k=0.7$ & \\
$N_{\min }=2$ & $n=2$ & $n=10$ & $37.4 \%$ \\
$N_{\max }=10$ & $E_{\mathrm{c}}=0.027 \mathrm{~J}$ & $E_{\mathrm{c}}=0.017 \mathrm{~J}$ & \\
\hline \hline$K_{\min }=2$ & $k=2$ & $k=2$ & \\
$N_{\min }=2$ & $n=2$ & $n=4$ & $7.9 \%$ \\
$N_{\max }=10$ & $E_{\mathrm{c}}=0.053 \mathrm{~J}$ & $E_{\mathrm{c}}=0.049 \mathrm{~J}$ & \\
\hline
\end{tabular}

Table V

VISUAL FEATURES EXTRACTION APPLICATION SCENARIO

\begin{tabular}{|c||c|c|c|}
\hline Constraints & Ad-hoc deployment & Proposed approach & Gain \\
\hline \hline$K_{\min }=1.25$ & $k=1.25$ & $k=1.25$ & \\
$N_{\min }=2$ & $n=2$ & $n=10$ & $30.8 \%$ \\
$N_{\max }=10$ & $E_{\mathrm{c}}=0.033 \mathrm{~J}$ & $E_{\mathrm{c}}=0.023 \mathrm{~J}$ & \\
\hline \hline$K_{\min }=2$ & $k=2$ & $k=2$ & \\
$N_{\min }=2$ & $n=2$ & $n=7$ & $18.1 \%$ \\
$N_{\max }=10$ & $E_{\mathrm{c}}=0.045 \mathrm{~J}$ & $E_{\mathrm{c}}=0.037 \mathrm{~J}$ & \\
\hline
\end{tabular}

\section{CONCLUSIONS}

We proposed an analytic model for the energy consumption of a uniformly-formed wireless visual sensor network (VSN) under varying spatio-temporal constraints, defined in terms of number of nodes to be deployed per network tier and video frames to be captured by each node. Analytic conditions for the optimal spatiotemporal settings within the VSN were derived for different probability density functions characterizing the multimedia data volume to be transmitted by each node. Monte-Carlo experiments performed via an energy-measurement testbed revealed that the proposed model's accuracy is within 7\% of the obtained energy consumption. Applying the model to two realistic scenarios for motion JPEG compression and local visual features extraction within each node in the VSN demonstrated that substantial energy savings can be obtained via the proposed approach against ad-hoc settings for the spatio-temporal parameters of the VSN. As such, the proposed model can be used for early-stage studies of VSNs to determine the best operational parameters to be considered prior to cumbersome and costly real-world deployment and testing.

\section{APPENDIX A}

We first present the detailed proof of Proposition 1 under the Uniform distribution $(\mathrm{D}=\mathrm{U})$. The proofs for the Pareto, Exponential and Half-Gaussian distributions (i.e., Proposition 2) are summarized afterward, since they follow the same steps as for the case of the Uniform.

\section{A. Proof of Proposition 1 for the Uniform Distribution}

1) Investigating the n-direction: We examine the function $E_{\mathrm{c}, \mathrm{U}}$ along the plane $k=\bar{k}, \bar{k} \geq K_{\min }$, and analyze $E_{\mathrm{c}, \mathrm{U}}(n, \bar{k})$ which is now a function of $n$ only. It is straightforward to show by first-derivative analysis that the only candidate extremum or inflection point of $E_{\mathrm{c}, \mathrm{U}}(n, \bar{k})$ is $n_{0, \mathrm{U}}=\frac{\beta_{\mathrm{U}}}{k}$, with $\beta_{\mathrm{U}}$ given by 18 . This candidate extremum holds under the assumption that: $N_{\min } \leq n_{0, \mathrm{U}} \leq N_{\max }$, i.e. that the candidate extremum or inflection point of $E_{\mathrm{c}, \mathrm{U}}(n, \bar{k})$ falls within the predefined spatial constraints of (7). Furthermore, we find that $\left.\frac{d^{2} E_{\mathrm{c}, \mathrm{U}}(n, \bar{k})}{d n^{2}}\right|_{n=n_{0, \mathrm{U}}}>0$, which demonstrates that $n_{0, \mathrm{U}}$ is a local minimum. Given that local extrema must alternate within the region of support of a continuous and differentiable function [45], $n_{0, \mathrm{U}}$ is also the global minimum of $E_{\mathrm{c}, \mathrm{U}}(n, \bar{k})$ within $N_{\min } \leq n \leq N_{\max }$.

Having derived the global minimum of $E_{\mathrm{c}, \mathrm{U}}(n, \bar{k})$ along an arbitrary plane $k=\bar{k}, \bar{k} \geq K_{\min }$, we can now attempt to find the value of $k, k \geq K_{\min }$, that minimizes the energy function. Evaluating $E_{\mathrm{c}, \mathrm{U}}(n, k)$ on $n=n_{0, \mathrm{U}}$, we obtain:

$$
\begin{aligned}
E_{\mathrm{c}, \mathrm{U}}\left(n_{0, \mathrm{U}}, k\right) & =k[a+r[(p+j)(d+1) \\
& \left.\left.-\frac{p^{2}(d+1)}{b+p}+h d+g\right]\right] .
\end{aligned}
$$

Evidently, the value of $k$ minimizing 26 is the minimum allowable, i.e. $k=K_{\min }$. Thus, the solution minimizing (6) in the $n$-direction is $\mathcal{S}_{n_{0, \mathrm{U}}}=\left(\frac{\beta_{\mathrm{U}}}{K_{\min }}, K_{\min }\right)$. This solution holds under the constraint:

$$
N_{\min } \leq \frac{\beta_{\mathrm{U}}}{K_{\min }} \leq N_{\max }
$$


2) Investigating the $k$-direction: Similarly, we cut $E_{\mathrm{c}, \mathrm{U}}(n, k)$ along the plane $n=\bar{n}, N_{\min } \leq \bar{n} \leq N_{\max }$, and minimize $E_{\mathrm{c}, \mathrm{U}}(\bar{n}, k)$ which is now a function of $k$ only. Following the steps presented earlier, we can show by first and second derivative analysis that the global minimum of $E_{\mathrm{c}, \mathrm{U}}(\bar{n}, k)$ occurs at $k_{0, \mathrm{U}}=\frac{\gamma_{\mathrm{U}}}{\bar{n}}$, with $\gamma_{\mathrm{U}}$ given by 19 . This global minimum holds under the assumption that $k_{0, \mathrm{U}} \geq K_{\min }$, due the predefined temporal constraint of (7). Having derived the global minimum of $E_{\mathrm{c}, \mathrm{U}}(\bar{n}, k)$ along an arbitrary plane $n=\bar{n}, N_{\min } \leq \bar{n} \leq N_{\max }$, we can now attempt to find the value of $n, N_{\min } \leq n \leq N_{\max }$, that minimizes the energy function. Evaluating $E_{\mathrm{c}, \mathrm{U}}(n, k)$ on $k=k_{0, \mathrm{U}}$ we obtain:

$$
\begin{aligned}
E_{\mathrm{c}, \mathrm{U}}\left(n, k_{0, \mathrm{U}}\right) & =\frac{1}{n}\left[[a+r[(p+j)(d+1)+h d+g]] \gamma_{\mathrm{U}}\right. \\
& \left.-p s+\frac{s^{2}(b+p)}{4 r(d+1) \gamma_{\mathrm{U}}}\right] .
\end{aligned}
$$

Evidently, the value of $n$ minimizing (28) is the maximum allowable, i.e. $n=N_{\max }$. Hence, the solution when attempting to minimize (28) in the $k$-direction under the constraints of (7) is $\mathcal{S}_{k_{0, \mathrm{U}}}=\left(N_{\max }, \frac{\gamma_{\mathrm{U}}}{N_{\max }}\right)$ under the constraint:

$$
K_{\min } \leq \frac{\gamma_{\mathrm{U}}}{N_{\max }} .
$$

3) Uniqueness of solution and solution when (27) and (29) do not hold: So far, we have found two solutions minimizing the energy consumption of each node: $\mathcal{S}_{n_{0, \mathrm{U}} \mathrm{U}}$, which minimizes the energy in the $n$-direction by appropriately choosing the number of nodes to deploy (spatial resolution), and $\mathcal{S}_{k_{0, \mathrm{U}}}$, which minimizes the energy in the $k$-direction by appropriately setting the optimal number of frames to capture (temporal resolution) during the active time interval. However, the following issues arise:

1) Both solutions are only applicable under constraints 27) and (29). Is it possible that both constraints are satisfied and, if so, then what is the best solution for (6)?

2) Conversely, if neither of these two constraints is satisfied, then what is the optimal solution for (6)?

It turns out that the answer to both questions can be derived based on the value of the temporal constraint, $K_{\min }$, as it is clarified in the following analysis.

Starting from (27), with a few straightforward manipulations we reach $\frac{\beta_{U}}{N_{\max }} \leq K_{\min } \leq \frac{\beta_{U}}{N_{\min }}$. The second constraint for $K_{\min }$ is provided by (29). It is now easy to prove that $\beta_{\mathrm{U}}>\gamma_{\mathrm{U}}$ (see Appendix B-A), which demonstrates that the constraints of the two solutions are non-overlapping, as the lower bound of 27) is larger than the upper bound of 29. This answers the first question.

To address the second question, we have to analyze what happens when $\frac{\gamma_{U}}{N_{\max }}<K_{\min }<\frac{\beta_{\mathrm{U}}}{N_{\max }}$ or $K_{\min }>\frac{\beta_{\mathrm{U}}}{N_{\min }}$, as neither of $\mathcal{S}_{n_{0, \mathrm{U}}}$ and $\mathcal{S}_{k_{0, \mathrm{U}}}$ are applicable in such cases. It is straightforward to show that $\frac{\partial E_{\mathrm{c}, \mathrm{U}}}{\partial n}$ and $\frac{\partial E_{\mathrm{c}, \mathrm{U}}}{\partial k}$ are never zero within these intervals. Hence, the solution we are looking for must lie on one of the two boundary points: $\left(N_{\min }, K_{\min }\right)$ or $\left(N_{\max }, K_{\min }\right)$.
Let us focus on the case of $\frac{\gamma_{\mathrm{U}}}{N_{\max }}<K_{\min }<\frac{\beta_{\mathrm{U}}}{N_{\max }}$ and evaluate $E_{\mathrm{c}, \mathrm{U}}(n, k)$ on the boundary plane $n=N_{\max }$. Since $E_{\mathrm{c}}\left(N_{\max }, k\right)$ is monotonically increasing for $k>\frac{\gamma_{\mathrm{U}}}{N_{\max }}$ the optimal point is $k=K_{\min }$, which leads to the solution $\mathcal{S}_{\max \min }=\left(N_{\max }, K_{\min }\right)$. Similarly, let us look at the $k$ direction by evaluating the energy function on the $k=K_{\min }$ plane. Now $n_{0, \mathrm{U}}=\frac{\beta_{\mathrm{U}}}{K_{\min }}$ is larger than $N_{\max }$ and is thus not admissible. Since $E_{\mathrm{c}, \mathrm{U}}\left(n, K_{\min }\right)$ is decreasing for $n<n_{0, \mathrm{U}}$, the optimal point is $n=N_{\max }$, which also leads to the solution $\mathcal{S}_{\max \text { min }}$. Finally, when $K_{\min }>\frac{\beta_{\mathrm{U}}}{N_{\min }}$, following a similar analysis we reach that the optimal solution is $\mathcal{S}_{\text {min min }}=\left(N_{\min }, K_{\min }\right)$.

Summarizing, when the data transmitted by each VSN node follows the Uniform distribution of (8), the set of solutions giving the minimum energy consumption in (6) under the spatio-temporal constraints of (7) is given by 17.

\section{B. Proof of Proposition 1 for the Pareto Distribution}

Considering the energy consumption for the Pareto distribution $E_{\mathrm{c}, \mathrm{P}}$ in (14), we follow the derivative-based analysis along each direction and join the obtained minima along with their constraints.

1) n-direction: The partial derivative of $E_{\mathrm{c}, \mathrm{P}}$ with respect to $n$ (i.e. under a plane $k=\bar{k}$ with $\bar{k} \geq K_{\min }$ ) is:

$$
\frac{\partial E_{\mathrm{c}, \mathrm{P}}}{\partial n}=-\frac{b s}{n^{2}}+\frac{s}{n^{2}}(b+p)\left(\frac{v n}{s}\right)^{\alpha} .
$$

The only solution for $\frac{\partial E_{\mathrm{c}, \mathrm{P}}}{\partial n}=0$ that can be admissible under the constraints of (7) is $n_{0, \mathrm{P}}=\frac{\beta_{\mathrm{P}}}{k}$, with $\beta_{\mathrm{P}}$ given by (21). It is straightforward to show that $n_{0, \mathrm{P}}$ corresponds to the global minimum of $E_{\mathrm{c}, \mathrm{P}}(n, \bar{k})$. Evaluating $E_{\mathrm{c}, \mathrm{P}}$ for $n_{0, \mathrm{P}}$ leads to

$$
\begin{aligned}
E_{\mathrm{c}, \mathrm{P}}\left(n_{0, \mathrm{P}}, k\right) & =\bar{k}[a+r[(j-b)(d+1)+h d+g \\
& \left.\left.+(d+1)(b)^{\frac{\alpha-1}{\alpha}}(b+p)^{\frac{1}{\alpha}}\right]\right]
\end{aligned}
$$

which attains its minimum value for the minimum allowable $\bar{k}$, i.e. at point $\mathcal{S}_{n_{0, \mathrm{P}}}=\left(\frac{\beta_{\mathrm{P}}}{K_{\min }}, K_{\text {min }}\right)$. Now we have to ensure that $N_{\min } \leq n_{0, \mathrm{P}} \leq N_{\max }$, which gives $\frac{\beta_{\mathrm{P}}}{N_{\max }} \leq K_{\min } \leq \frac{\beta_{\mathrm{P}}}{N_{\min }}$. As discussed for the Uniform case, for values of $K_{\min }$ outside this range, the optimal solution comprises the border points $\left(N_{\max }, K_{\min }\right)$ or $\left(N_{\min }, K_{\min }\right)$, depending on temporal constraint.

2) k-direction: The partial derivative of $E_{\mathrm{c}, \mathrm{P}}$ with respect to $k$ (i.e. under a plane $n=\bar{n}$ with $N_{\min } \leq \bar{n} \leq N_{\max }$ ) is:

$$
\begin{aligned}
\frac{\partial E_{\mathrm{c}, \mathrm{P}}}{\partial k} & =a+r[(j+p)(d+1)+h d+g] \\
& +r(b+p)(d+1) \\
& \times\left[\left(\frac{\bar{n}}{s}\right)^{\alpha-1}\left(\frac{k r(\alpha-1)(d+1)}{\alpha}\right)^{\alpha-1}-1\right] .
\end{aligned}
$$

The only solution for $\frac{\partial E_{\mathrm{c}, \mathrm{P}}}{\partial k}=0$ that is admissible under the constraints of (7) is $k_{0, \mathrm{P}}=\frac{\gamma_{\mathrm{P}}}{\bar{n}}$, with $\gamma_{\mathrm{P}}$ defined in (20). 
The first constraint imposed on $k_{0, \mathrm{P}}$ is that it must be positive, which leads to

$$
b>j+\frac{a}{r(d+1)}+\frac{h d+g}{d+1} .
$$

The last equation indicates that the global minimum of $k_{0, \mathrm{P}}$ holds only if the energy consumption during the idle state is greater than the energy during transmission. While this is possible from a mathematical point of view, the physical reality of wireless transceivers does not allow for this case to manifest in a practical setting. We also note that, beyond the constraint of (33), the global minimum of $k_{0, \mathrm{P}}$ holds under the assumption that $k_{0, \mathrm{P}} \geq K_{\min }$ due the predefined temporal constraint of (7).

Evaluating $E_{\mathrm{c}, \mathrm{P}}(n, k)$ on $k=k_{0, \mathrm{P}}$, we obtain

$$
\begin{aligned}
E_{\mathrm{c}, \mathrm{P}}\left(n, k_{0, \mathrm{P}}\right) & =\frac{b+p}{n}\left[s^{1-\alpha}\left(\frac{\beta_{\mathrm{P}} r(\alpha-1)(d+1)}{\alpha}\right)^{\alpha}\right. \\
& \left.\times(\alpha-1)^{-1}-\beta_{\mathrm{P}} r(d+1)\right] \\
& +\frac{b s}{n}+\frac{\gamma_{\mathrm{P}}}{N_{\max }} \frac{\beta_{\mathrm{P}}[a+r[(j+p)(d+1)+h d+g]]}{n}
\end{aligned}
$$

Evidently, for $\alpha>1$, the value of $n$ minimizing (34) is the maximum allowable, i.e. $n=N_{\max }$. Hence, the solution when attempting to minimize the energy consumption function in the $k$-direction under the constraints of (7) is $\mathcal{S}_{k_{0, \mathrm{P}}}=\left(N_{\max }, \frac{\gamma_{\mathrm{P}}}{N_{\max }}\right)$ under the constraint $K_{\min } \leq \frac{\gamma_{\mathrm{P}}}{N_{\max }}$. It is now easy to prove that $\beta_{\mathrm{P}}>\gamma_{\mathrm{P}}$ (see Appendix B-B), which demonstrates that the constraints of the two solutions are non-overlapping.

\section{Exponential Distribution}

The energy consumption in the case of Exponential distribution is $E_{\mathrm{c}, \mathrm{E}}$, given by (15). We follow the derivativebased analysis along each direction and join together the obtained minima along with their constraints.

1) n-direction: The partial derivative of $E_{\mathrm{c}, \mathrm{E}}$ with respect to $n$ (i.e. under a plane $k=\bar{k}$ with $\bar{k} \geq K_{\min }$ ) is:

$$
\frac{\partial E_{\mathrm{c}, \mathrm{E}}}{\partial n}=-\frac{b s}{n^{2}}+\frac{s}{n^{2}}(b+p) \exp \left(-\frac{s}{n k r(d+1)}\right),
$$

which, under the constraints of 7 is equal to zero for $n_{0, \mathrm{E}}=$ $\frac{\beta_{\mathrm{E}}}{k}$, with $\beta_{\mathrm{E}}$ given by 22 . It is straightforward to show that $n_{0, \mathrm{E}}$ corresponds to the global minimum of $E_{\mathrm{c}, \mathrm{E}}(n, \bar{k})$. Evaluating $E_{\mathrm{c}, \mathrm{E}}$ for $n_{0, \mathrm{E}}$ leads to:

$$
\begin{aligned}
E_{\mathrm{c}, \mathrm{E}}\left(n_{0, \mathrm{E}}, \bar{k}\right) & =\bar{k}[a+r[j(d+1)+h d+g \\
& \left.\left.+b(d+1) \ln \left(\frac{b+p}{b}\right)\right]\right],
\end{aligned}
$$

which has its minimum value for the minimum allowable $\bar{k}$, i.e. at point $\mathcal{S}_{n_{0, \mathrm{E}}}=\left(\frac{\beta_{\mathrm{E}}}{K_{\text {min }}}, K_{\text {min }}\right)$. Now we have to ensure that $N_{\min } \leq n_{0, \mathrm{E}} \leq N_{\max }$, which leads to $\frac{\beta_{\mathrm{E}}}{N_{\max }} \leq K_{\min } \leq \frac{\beta_{\mathrm{E}}}{N_{\min }}$. Again, for values of $K_{\min }$ outside this range, the optimal solution comprises the border points $\left(N_{\max }, K_{\min }\right)$ or $\left(N_{\min }, K_{\min }\right)$, depending on temporal constraint.
2) k-direction: The partial derivative of $E_{\mathrm{c}, \mathrm{E}}$ with respect to $k$ (i.e. under a plane $n=\bar{n}$ with $N_{\min } \leq \bar{n} \leq N_{\max }$ ) is:

$$
\begin{gathered}
\frac{\partial E_{\mathrm{c} . \mathrm{E}}}{\partial k}=[a+r[(p+j)(d+1)+h d+g]] \\
+(b+p)\left[r(d+1)\left(\exp \left(-\frac{s}{\bar{n} k r(d+1)}\right)-1\right)\right. \\
\left.+\frac{s}{\bar{n} k} \exp \left(-\frac{s}{\bar{n} k r(d+1)}\right)\right] .
\end{gathered}
$$

The only solution for $\frac{\partial E_{\text {c.E }}}{\partial k}=0$ that may be admissible under the constraints of $(7)$ is $k_{0, \mathrm{E}}=\frac{\gamma_{\mathrm{E}}}{\bar{n}}$, with $\gamma_{\mathrm{E}}$ defined in (23).

The first constraint imposed on $k_{0, \mathrm{E}}$ is that it must be positive. That is, the product-log function should be smaller than -1 . This is true when the argument of the product-log function is limited within $\left(-\frac{1}{\exp }, 0\right)[33$. That is:

$$
-\frac{1}{\exp }<\frac{a-r[(b-j)(d+1)-h d-g]}{\exp \times r(d+1)(b+p)}<0 .
$$

It is easy to verify that a necessary condition for (38) to hold is (33). Thus, similar to the Pareto case, while the the global minimum of $k_{0, \mathrm{E}}$ is in principle possible, it is not expected to be encountered in a practical setup. Beyond the constraint of (38), the global minimum of $k_{0, \mathrm{E}}$ holds under the assumption that $k_{0, \mathrm{E}} \geq K_{\min }$ due the predefined temporal constraint of (7).

Having derived the global minimum of $E_{\mathrm{c}, \mathrm{E}}(\bar{n}, k)$ along an arbitrary plane $n=\bar{n}, N_{\min } \leq \bar{n} \leq N_{\max }$, we can now attempt to find the value of $n, N_{\min } \leq n \leq N_{\max }$, that minimizes the energy function. Evaluating $E_{\mathrm{c}, \mathrm{E}}(n, k)$ on $k=k_{0, \mathrm{P}}$ we obtain

$$
\begin{aligned}
E_{\mathrm{c}, \mathrm{E}}\left(n, k_{0, \mathrm{P}}\right) & =\frac{r \gamma_{\mathrm{E}}(b+p)(d+1)}{n}\left(\exp \left(-\frac{1}{r \gamma_{\mathrm{E}}(d+1)}\right)-1\right) \\
& +\frac{b s}{n}+\frac{\gamma_{\mathrm{E}}(a+r[(p+j)(d+1)+h d+g]}{n} .
\end{aligned}
$$

Evidently, for $\alpha>1$, the value of $n$ minimizing (39) is the maximum allowable, i.e. $n=N_{\max }$. Hence, the solution when attempting to minimize the energy consumption function in the $k$-direction under the constraints of $(7)$ is $\mathcal{S}_{k_{0, \mathrm{P}}}=\left(N_{\max }, \frac{\gamma_{\mathrm{E}}}{N_{\max }}\right)$ under the constraint $K_{\min } \leq \frac{\gamma_{\mathrm{E}}}{N_{\max }}$. It is now easy to prove that $\beta_{\mathrm{E}}>\gamma_{\mathrm{E}}$ (see Appendix B-B), which demonstrates that the constraints of the two solutions are non-overlapping.

\section{Half-Gaussian Distribution}

The energy consumption for half-Gaussian distribution is $E_{\mathrm{c}, \mathrm{H}}$ given by (16).

1) n-direction: The partial derivative of $E_{\mathrm{c}, \mathrm{H}}$ with respect to $n$ (i.e. under a plane $k=\bar{k}$ with $\bar{k} \geq K_{\min }$ ) is:

$$
\frac{\partial E_{\mathrm{c}, \mathrm{H}}}{\partial n}=\frac{p s}{n^{2}}-\frac{s(b+p)}{n^{2}} \operatorname{erf}\left(\frac{s}{\sqrt{\pi} \bar{k} r n(d+1)}\right),
$$

which, under the constraints of 7, is equal to zero for $n_{0, \mathrm{H}}=\frac{\beta_{\mathrm{H}}}{k}$, with $\beta_{\mathrm{H}}$ given by 25]. It is easy to show that 
$n_{0, \mathrm{H}}$ corresponds to the global minimum of $E_{\mathrm{c}, \mathrm{H}}(n, \bar{k})$. Evaluating $E_{\mathrm{c}, \mathrm{H}}$ for $n_{0, \mathrm{H}}$ leads to:

$$
\begin{aligned}
E_{\mathrm{c}, \mathrm{H}}\left(n_{0, \mathrm{H}}, k\right) & =k[a+r[(p+j)(d+1)+h d+g \\
& \left.\left.+(b+p)(d+1) \exp \left(\left[-\operatorname{erf}^{-1}\left(\frac{p}{b+p}\right)\right]^{2}\right)\right]\right] .
\end{aligned}
$$

which has its minimum value for the minimum allowable $\bar{k}$, i.e. at $\mathcal{S}_{n_{0, \mathrm{H}}}=\left(\frac{\beta_{\mathrm{H}}}{K_{\min }}, K_{\text {min }}\right)$. Now, we have to ensure that $N_{\min } \leq n_{0, \mathrm{H}} \leq N_{\max }$, which leads to $\frac{\beta_{\mathrm{H}}}{N_{\max }} \leq K_{\min } \leq \frac{\beta_{\mathrm{H}}}{N_{\min }}$. Similarly as for the previous distributions, for values of $K_{\text {min }}$ outside this range, the optimal solution comprises the border points $\left(N_{\max }, K_{\min }\right)$ or $\left(N_{\min }, K_{\min }\right)$.

2) $k$-direction: The partial derivative of $E_{\mathrm{c}, \mathrm{H}}$ with respect to $k$ (i.e. under a plane $n=\bar{n}$ with $N_{\min } \leq \bar{n} \leq N_{\max }$ ) is:

$$
\begin{aligned}
\frac{\partial E_{\mathrm{c}, \mathrm{H}}}{\partial k} & =[a+r[(p+j)(d+1)+h d+g] \\
& +r(b+p)(d+1) \\
& \times\left(\exp \left(-\frac{s^{2}}{\pi k^{2} r^{2} \bar{n}^{2}(d+1)^{2}}\right)-1\right),
\end{aligned}
$$

which can be shown to be positive. Hence, the energy function is increasing with respect to $k$ and the optimal value is the minimum allowable $k$. Thus, the solution is equal to $\mathcal{S}_{n_{0, \mathrm{H}}}$.

\section{APPENDIX B}

\section{A. Proof that $\beta_{U}>\gamma_{U}$}

Replacing $\beta_{U}$ and $\gamma_{U}$ from (18) and (19) in the inequality we desire to prove, squaring both sides (since all terms are positive) and rearranging terms, leads to

$$
\begin{aligned}
r[b(g+j+p)+p(g+ & j+p d)+b d(h+j+p) \\
& +p d(h+j)]+a(b+p)>0,
\end{aligned}
$$

which is indeed positive because all constants are positive quantities.

\section{B. Proof that $\beta_{P}>\gamma_{P}$}

Replacing the terms $\beta_{\mathrm{P}}$ and $\gamma_{\mathrm{P}}$ from (20) and (21) in the inequality we desire to prove, we reach:

$$
\left(\frac{b}{b+p}\right)^{\frac{1}{\alpha}}>\left(\frac{-a+r[(b-j)(d+1)-h d-g]}{r(d+1)(b+p)}\right)^{\frac{1}{\alpha-1}} .
$$

Now, recalling the constraint of (33), let us assume the minimum possible value for $b$, i.e.,

$$
b=j+\frac{a}{r(d+1)}+\frac{h d+g}{d+1}+\delta
$$

with $\delta>0$. Evidently, $b>\delta$ since all constants are positive. Substituting $b$ in the numerator of the right hand side of (44) via 45, we obtain $\left(\frac{b}{b+p}\right)^{\frac{1}{\alpha}}>\left(\frac{\delta}{b+p}\right)^{\frac{1}{\alpha-1}}$. Since $b>\delta$, in order to prove the last expression it suffices to prove that $\left(\frac{b}{b+p}\right)^{\frac{1}{\alpha}}>\left(\frac{b}{b+p}\right)^{\frac{1}{\alpha-1}}$ holds. The last expression is indeed true because $\frac{b}{b+p} \leq 1$.

\section{Proof that $\beta_{E}>\gamma_{E}$}

Replacing $\beta_{\mathrm{E}}$ and $\gamma_{\mathrm{E}}$ from (23) and 22, in the inequality we desire to prove, we reach

$$
\frac{1}{\ln \left(\frac{b+p}{p}\right)}>-\frac{1}{W\left(\frac{a-r[(b-j)(d+1)-h d-g]}{\exp \times r(d+1)(b+p)}\right)+1} .
$$

Recalling that, under the constraint (33), the Lambert $W$ function is upper-bounded by -1 we obtain

$$
-\ln \left(\frac{b+p}{p}\right)-1>W\left(\frac{a-r[(b-j)(d+1)-h d-g]}{\exp \times r(d+1)(b+p)}\right) .
$$

Substituting $b$ in the numerator of the right side of 47 with the expression of 45 and using the definition of the product-log function, $z=W(z) \exp (W(z))$, the last inequality leads to $p>\frac{-\delta}{W\left(\frac{-\delta}{\exp \times(b+p)}\right)}$. The right-hand side is upper bounded by $\delta$, since the Lambert function is upper bounded by -1 . Thus, to complete the proof, it suffices to prove that $p>\delta$. For derivating the solutions in the Exponential case, we have assumed that $p>b$ and 45. shows that $b>\delta$. Therefore, $p>\delta$.

\section{REFERENCES}

[1] V. P. Munishwar and N. B. Abu-Ghazaleh, "Coverage algorithms for visual sensor networks," ACM Trans. on Sensor Netw., vol. 9, no. 4, p. 45,2013

[2] X. Wang and H. Qian, "Constructing a 6LoWPAN wireless sensor network based on a cluster tree," IEEE Trans. on Vehicular Technology, vol. 61, no. 3, pp. 1398-1405, Mar. 2012.

[3] A. Tinka, T. Watteyne, and K. Pister, "A decentralized scheduling algorithm for time synchronized channel hopping," Lect. Notes Inst. Comp. Sci. (Ad-Hoc Networks), vol. 49, no. 4, pp. 201-216, Aug. 2010 .

[4] M. Zorzi, A. Gluhak, S. Lange, and A. Bassi, "From today's INTRAnet of things to a future INTERnet of things: a wirelessand mobility-related view," IEEE Wireless Comm., vol. 17, no. 6, pp. 44-51, Dec. 2010.

[5] J. Gubbi, R. Buyya, S. Marusic, and M. Palaniswami, "Internet of Things (IoT): A vision, architectural elements, and future directions," Future Gen. Comp. Syst. J., vol. 29, no. 7, pp. 1645-1660, Sep. 2013.

[6] S. Ehsan and B. Hamdaoui, "A survey on energy-efficient routing techniques with qos assurances for wireless multimedia sensor networks," IEEE Comm. Surv \& Tut., vol. 14, no. 2, pp. 265-278, 2012.

[7] D. Buranapanichkit and Y. Andreopoulos, "Distributed timefrequency division multiple access protocol for wireless sensor networks," IEEE Wireless Comm. Letters, vol. 1, no. 5, pp. 440 443, Oct. 2012.

[8] Z. Zuo, Q. Lu, and W. Luo, "A two-hop clustered image transmission scheme for maximizing network lifetime in wireless multimedia sensor networks," J. Comput. Commun., vol. 35, no. 1, pp. 100-108, 2012.

[9] S. Pudlewski, A. Prasanna, and T. Melodia, "Compressed-sensingenabled video streaming for wireless multimedia sensor networks," IEEE Trans. Mobile Comput., vol. 11, no. 6, pp. 1060-1072, 2012.

[10] J. F. Mingorance-Puga, G. Maciá-Fernández, A. Grilo, and N. M. Tiglao, "Efficient multimedia transmission in wireless sensor networks," in IEEE Proc. 6th EURO-NF Conf. on Next Gen. Internet (NGI), 2010, pp. 1-8.

[11] A. Newell and K. Akkaya, "Self-actuation of camera sensors for redundant data elimination in wireless multimedia sensor networks," in Proc. IEEE Internat. Conf. Comm., ICC'09. IEEE, 2009, pp. $1-5$.

[12] A. Canclini, L. Baroffio, M. Cesana, A. Redondi, and M. Tagliasacchi, "Comparison of two paradigms for image analysis in visual sensor networks," in Proceedings of the 11th ACM Conference on Embedded Networked Sensor Systems, ser. SenSys '13. ACM, 2013, pp. 62:1-62:2. 
[13] P. Chen, P. Ahammad, C. Boyer, S.-I. Huang, L. L., E. Lobaton, M. Meingast, S. Oh, S. Wang, P. Yan, A. Yang, C. Yeo, L.-C. Chang, J. D. Tygar, and S. Sastry, "CITRIC: A low-bandwidth wireless camera network platform," in ACM/IEEE Int. Conf. on Distrib. Smart Cam., ICDSC, Sept., pp. 1-10.

[14] M. Rahimi, R. Baer, O. I. Iroezi, J. C. Garcia, J. Warrior, D. Estrin, and M. Srivastava, "Cyclops: in situ image sensing and interpretation in wireless sensor networks," in Proc. ACM Internat. Conf. on Embed. Netw. Sensor Syst. ACM, 2005, pp. 192-204.

[15] S. Hengstler, D. Prashanth, S. Fong, and H. Aghajan, "MeshEye: a hybrid-resolution smart camera mote for applications in distributed intelligent surveillance," in Proc. IEEE Internat. Symp. on Inf Process. in Sensor Net.IPSN 2007. IEEE, 2007, pp. 360-369.

[16] D. G. Lowe, "Distinctive image features from scale-invariant keypoints," Internat. J. of Comput. Vision, vol. 60, no. 2, pp. 91-110, 2004.

[17] A. Redondi, L. Baroffio, M. Cesana, and M. Tagliasacchi, "Compress-then-analyze vs analyze-then-compress. two paradigms for image analysis in visual sensor networks." in MMSP, 2013.

[18] A. Bachir, M. Dohler, T. Watteyne, and K. Leung, "MAC essentials for wireless sensor networks," IEEE Comm. Surv. Tut., vol. 12, no. 2, pp. $222-248$, quarter 2010 .

[19] M. Tahir and R. Farrell, "A cross-layer framework for optimal delaymargin, network lifetime and utility tradeoff in wireless visual sensor networks," Ad Hoc Networks, vol. 11, no. 2, pp. 701 - 711, 2013.

[20] D. Kandris, M. Tsagkaropoulos, I. Politis, A. Tzes, and S. Kotsopoulos, "Energy efficient and perceived QoS aware video routing over wireless multimedia sensor networks," Ad Hoc Networks, vol. 9, no. 4, pp. 591-607, 2011

[21] V. P. Munishwar and N. B. Abu-Ghazaleh, "Coverage algorithms for visual sensor networks," ACM Trans. Sen. Netw., vol. 9, no. 4, pp 45:1-45:36, Jul. 2013.

[22] A. Koubaa, A. Cunha, and M. Alves, "A time division beacon scheduling mechanism for IEEE 802.15.4/zigbee cluster-tree wireless sensor networks," in 19th Euromicro Conf. on Real-Time Syst. ECRTS '07, july 2007, pp. $125-135$.

[23] J. Degesys, I. Rose, A. Patel, and R. Nagpal, "DESYNC: selforganizing desynchronization and TDMA on wireless sensor networks," in Proc. 6th Int. Conf. on Inf. Process. in Sensor Netw. IPSN, 2007, pp. 11-20.

[24] E. Y. Lam and J. W. Goodman, "A mathematical analysis of the DCT coefficient distributions for images," IEEE Trans. on Image Processing, vol. 9, no. 10, pp. 1661-1666, Oct. 2000

[25] B. Foo, Y. Andreopoulos, and M. van der Schaar, "Analytical rate-distortion-complexity modeling of wavelet-based video coders," IEEE Trans. on Signal Processing, vol. 56, no. 2, pp. 797-815, Feb. 2008.

[26] I. F. Akyildiz, T. Melodia, and K. R. Chowdhury, "Wireless Multimedia Sensor Networks: Applications and Testbeds," Proc. of the IEEE, vol. 96, no. 10, pp. 1588-1605, Oct. 2008.

[27] V. Paxson and S. Floyd, "Wide-area traffic: The failure of Poisson modeling," IEEE/ACM Trans. on Networking, pp. 226-244, 1995.

[28] K. Park, "On the relationship between file sizes, transport protocols, and self-similar network traffic," in Proc. IEEE Internat. Conf. on Network Protocols, ICNP, 1996, pp. 171-180.
[29] R. Kumar, "A protocol with transcoding to support QoS over internet for multimedia traffic," in IEEE Proc. of Internat. Conf. on Multimedia and Expo, ICME, ser. ICME '03. Washington, DC, USA: IEEE Computer Society, 2003, pp. 465-468.

[30] M. Dai, Z. Yueping, and D. Loguinov, "A unified traffic model for MPEG-4 and H.264 video traces," IEEE Trans. on Multimedia, vol. 11, no. 5, pp. 1010-1023, Aug. 2009.

[31] M. Tagliasacchi, S. Tubaro, and A. Sarti, "On the modeling of motion in Wyner-Ziv video coding," in Proc. IEEE Internat. Conf. on Image Process., ICIP, 2006, pp. 593-596.

[32] Y. Andreopoulos and I. Patras, "Incremental refinement of image salient-point detection," IEEE Trans. on Image Processing, vol. 17, no. 9, pp. 1685-1699, Sept. 2008.

[33] R. M. Corless, G. H. Gonnet, D. E. Hare, D. J. Jeffrey, and D. E. Knuth, "On the lambertw function," Advances in Computational mathematics, vol. 5, no. 1, pp. 329-359, 1996.

[34] A. Koubâa, M. Alves, and E. Tovar, "GTS allocation analysis in IEEE 802.15.4 for real-time wireless sensor networks," in IEEE Proc. Internat. Par. and Distrib. Process. Symp., IPDPS. IEEE, 2006, pp. 8-pp.

[35] "IEEE standard for local and metropolitan area networks-part 15.4: Low-rate wireless personal area networks (LR-WPANs) amendment 1: MAC sublayer,' IEEE Std 802.15.4e-2012 (Amendment to IEEE Std 802.15.4-2011), pp. 1-225, April 2012.

[36] P. Yi, A. Iwayemi, and C. Zhou, "Developing ZigBee deployment guideline under WiFi interference for smart grid applications," IEEE Trans. on Smart Grid, vol. 2, no. 1, pp. 110-120, March 2011.

[37] K. Pister and L. Doherty, "TSMP: Time synchronized mesh protocol," IASTED Distributed Sensor Networks, pp. 391-398, 2008.

[38] Y. Kwon and D. Shin, "The security monitoring system using IEEE 802.15.4 Protocol and CMOS Image Sensor," in Proc. IEEE Internat. Conf. on New Trends in Inf. and Serv. Sci., NISS '09, pp. 1197-1202.

[39] S. Paniga, L. Borsani, A. Redondi, M. Tagliasacchi, and M. Cesana, "Experimental evaluation of a video streaming system for wireless multimedia sensor networks," in IEEE/IFIP Annual Ad Hoc Netw. Worksh. IEEE, 2011, pp. 165-170.

[40] A. Redondi, M. Cesana, and M. Tagliasacchi, "Rate-accuracy optimization in visual wireless sensor networks," in Proc. IEEE Internat. Conf. Image Process., ICIP. IEEE, 2012, pp. 1105-1108.

[41] W. Yu, Z. Sahinoglu, and A. Vetro, "Energy efficient JPEG 2000 image transmission over wireless sensor networks," in Proc. IEEE Global Telecom. Conf., GLOBECOM, vol. 5. IEEE, 2004, pp. 2738 2743.

[42] E. Rosten, R. Porter, and T. Drummond, "FASTER and better: A machine learning approach to corner detection," IEEE Trans. Patt. Anal. and Machine Intel., vol. 32, pp. 105-119, 2010.

[43] M. Calonder, V. Lepetit, C. Strecha, and P. Fua, "Brief: Binary robust independent elementary features," Proc. IEEE Internat. Conf. on Comput. Vision, ECCV, pp. 778-792, 2010.

[44] W. R. Gilks and P. Wild, "Adaptive rejection sampling for Gibbs sampling," Applied Stat., pp. 337-348, 1992.

[45] G. H. Hardy, A Course of Pure Mathematics Centenary Edition. Cambridge University Press, 2008. 


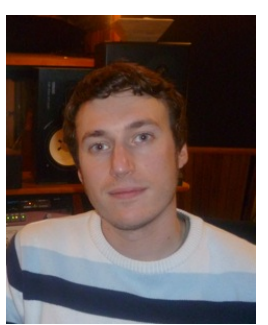

Alessandro Redondi received his Master degree in Computer Engineering in July 2009 and the Ph.D. in Information Engineering in 2014, both from Politecnico di Milano. Currently he is a post-doctoral researcher at the Department of Electronics and Information of Politecnico di Milano. His research activities are focused on algorithms and protocols for Visual Sensor Networks and Real Time Localization Systems.

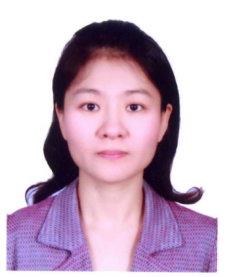

Dujdow Buranapanichkit is Lecturer in the Department of Electrical Engineering, Faculty of Engineering, Prince of Songkla University (Thailand). She received her $\mathrm{PhD}$ from the Department of Electronic and Electrical Engineering of University College London (UK). Her research interests are in wireless sensor networks and distributed synchronization mechanisms and protocol design.

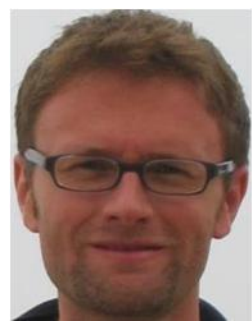

Matteo Cesana is currently an Assistant Professor with the Dipartimento di Elettronica, Informazione e Bioingegneria of the Politecnico di Milano, Italy. He received his MS degree in Telecommunications Engineering and his Ph.D. degree in Information Engineering from Politecnico di Milano in July 2000 and in September 2004, respectively. From September 2002 to March 2003 he was a visiting researcher at the Computer Science Department of the University of California in Los Angeles (UCLA). His research activities are in the field of design, optimization and performance evaluation of wireless networks with a specific focus on wireless sensor networks and cognitive radio networks. Dr. Cesana is an Associate Editor of the Ad Hoc Networks Journal (Elsevier).

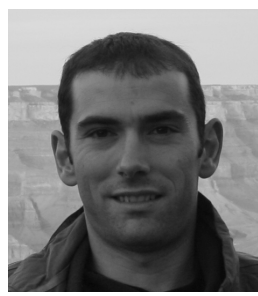

Marco Tagliasacchi is currently Assistant Professor at the "Dipartimento di Elettronica e Informazione - Politecnico di Milano", Italy. He received the "Laurea" degree (2002, cum Laude) in Computer Engineering and the Ph.D. in Electrical Engineering and Computer Science (2006), both from Politecnico di Milano. $\mathrm{He}$ was visiting academic at the Imperial College London (2012) and visiting scholar at the University of California, Berkeley (2004). His research interests include multimedia forensics, multimedia communications (visual sensor networks, coding, quality assessment) and information retrieval. Dr. Tagliasacchi co-authored more than 120 papers in international journals and conferences, including award winning papers at MMSP 2013, MMSP2012, ICIP 2011, MMSP 2009 and QoMex 2009. He has been actively involved in several EUfunded research projects. He is currently co-coordinating two ICT-FP7 FET-Open projects (GreenEyes - www.greeneyesproject.eu, REWIND www.rewindproject.eu). Dr. Tagliasacchi is an elected member of the IEEE Information Forensics and Security Technical committee for the term 2014-2016, and served as member of the IEEE MMSP Technical Committee for the term 2009-2012. He is currently Associate Editor for the IEEE Transactions on Circuits and Systems for Video Technologies (2011 best AE award) and APSIPA Transactions on Signal and Information Processing. Dr. Tagliasacchi was General co-Chair of IEEE Workshop on Multimedia Signal Processing (MMSP 2013, Pula, Italy) and he will be Technical Program Coordinator of IEEE International Conference on Multimedia\&Expo (ICME 2015, Turin, Italy).

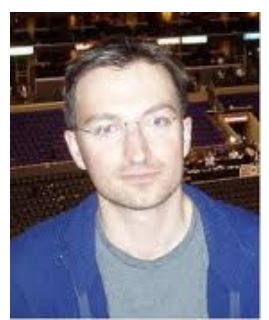

Yiannis Andreopoulos (M00) is Senior Lecturer in the Department of Electronic and Electrical Engineering of University College London (UK). His research interests are in wireless sensor networks, error-tolerant computing and multimedia systems. 